\title{
Development
}

\section{Swimming Exercise Promotes Post-injury Axon Regeneration and Functional Restoration through AMPK}

\author{
Sandeep Kumar, ${ }^{1, *}$ Sibaram Behera, ${ }^{1, *}$ Atrayee Basu, ${ }^{1}$ Shirshendu Dey, ${ }^{2}$ and ${ }^{D}$ Anindya Ghosh-Roy ${ }^{1}$
}

https://doi.org/10.1523/ENEURO.0414-20.2021

${ }^{1}$ Department of Biotechnology National Brain Research Centre, Manesar 122052, India and ${ }^{2}$ Bruker India Scientific Pvt. Ltd, New Delhi 110019, India

\begin{abstract}
Restoration of lost function following a nervous system injury is limited in adulthood as the regenerative capacity of nervous system declines with age. Pharmacological approaches have not been very successful in alleviating the consequences of nervous system injury. On the contrary, physical activity and rehabilitation interventions are often beneficial to improve the health conditions in the patients with neuronal injuries. Using touch neuron circuit of Caenorhabditis elegans, we investigated the role of physical exercise in the improvement of functional restoration after axotomy. We found that a swimming session of 90 min following the axotomy of posterior lateral microtubule (PLM) neuron can improve functional recovery in larval and adult stage animals. In older age, multiple exercise sessions were required to enhance the functional recovery. Genetic analysis of axon regeneration mutants showed that exercise-mediated enhancement of functional recovery depends on the ability of axon to regenerate. Exercise promotes early initiation of regrowth, self-fusion of proximal and distal ends, as well as postregrowth enhancement of function. We further found that the swimming exercise promotes axon regeneration through the activity of cellular energy sensor AAK-2/AMPK in both muscle and neuron. Our study established a paradigm where systemic effects of exercise on functional regeneration could be addressed at the single neuron level.
\end{abstract}

Key words: C. elegans; axotomy; PLM neuron; swimming exercise; axon regeneration; AAK-2

\section{Significance Statement}

Accelerating axonal regeneration and subsequent functional restoration is a major challenge to the people with nervous system injury. Research on rodents and humans suggests that rehabilitation therapy helps regain the lost function after neuronal injury. The nematode Caenorhabditis elegans provides an advantage to investigate the role of exercise in facilitating the axonal regeneration at the level of single neuron. Our study shows that swimming exercise promotes functional restoration via structural and functional changes in injured mechanosensory neuron. The benefit of exercise in regeneration depends on the metabolic energy sensor AAK-2/AMPK. This study provides a molecular perspective to exercise-mediated enhancement of axon regeneration.

\section{Introduction}

Neuronal injuries are accompanied by physical disruption of the axons, which leads to the loss of sensory or motor function (Johnson et al., 2013; Yang et al., 2015).

Received September 24, 2020; accepted April 25, 2021; First published May 24, 2021.

The authors declare no competing financial interests.
CNS has limited capacity to regenerate because of intrinsic failure and several inhibitory factors in the environment (Huebner and Strittmatter, 2009; He and Jin, 2016; van Niekerk et al., 2016). In case of peripheral nervous

Author contributions: S.K. and A.G.-R. designed research; S.K., S.B., and A.B. performed research; S.D. contributed unpublished reagents/analytic tools; S.K., S.B., and A.B. analyzed data; S.K. and A.G.-R. wrote the paper. 
system, regeneration from the injured proximal stump and subsequent growth toward the target tissues can lead to functional restoration ( $\mathrm{He}$ and Jin, 2016; Laha et al., 2017). However, this capability declines with age, resulting in partial or no functional restoration (Verdú et al., 2000; Geoffroy et al., 2016; Abay et al., 2017; Basu et al., 2017). Indeed, intrinsic capacity of axonal regrowth declines with age (Verdú et al., 2000) and external microenvironment poses various challenges in long distance axon regrowth (Brosius Lutz and Barres, 2014).

Although there is comprehensive understanding of axon regeneration pathways ( $\mathrm{He}$ and Jin, 2016; Mahar and Cavalli, 2018; Richardson and Shen, 2019), protective measures against aging-related loss of regenerative potential are rather lacking. On the contrary, accumulating evidences suggest a beneficial role of rehabilitation therapies and physical exercise in promoting functional recovery following spinal cord and other injuries in human (van Hedel and Dietz, 2010; Formento et al., 2018). Physical exercise enhances functional restoration after nervous system injury in primates and rat models as well (Capogrosso et al., 2016; Fu et al., 2016). Electrical stimulation and modulation of central pattern generator leads to dramatic increase in locomotor activity in humans and rats after spinal cord lesion (Wagner et al., 2018). The benefit of exercise is accompanied by remodeling of various parts of brain (Karssemeijer et al., 2017; Horowitz et al., 2020). Although many evidence suggest that exercise promotes regrowth of injured axon in peripheral system (Park and Höke, 2014; Gordon and English, 2016; Chen et al., 2017b), it is not completely clear whether functional improvement is the outcome of rewiring of injured axon or remodeling of spared circuitry. Also, cellular and molecular mechanisms involving exercise-mediated functional improvement after nerve injury are not clear.

Caenorhabditis elegans is an excellent model to investigate the cellular and molecular mechanism of axon regeneration following laser-assisted injury (Hammarlund et al., 2009; He and Jin, 2016; Richardson and Shen, 2019). Axonal injury leads to calcium influx and activates the conserved p38-MAPK pathway involving dual leucine zipper kinase (DLK)-1, which initiates the transcription of regeneration associated genes (Hammarlund et al., 2009;

This work is supported by the National Brain Research Centre core fund from the Department of Biotechnology, The India Alliance Department of Biotechnology Wellcome Grant IA/I/13/1/500874, and the Science and Engineering Research Board (SERB) Grant CRG/2019/002194. S.K. was supported by the SERB-National Post-doctoral Fellowship scheme (NPDF) SERB File Number PDF/2017/001610.

${ }^{*}$ S.K. and S.B. contributed equally to this work.

Acknowledgements: We thank NBRP, Japan and Caenorhabditis Genetics Center (CGC) for strains; Devashish Pandey for helping with thrashing frequency analysis; Kavita Babu and Swagata Dey for the comments on this manuscript; and A. Mukhopadhyay and S. Koushika for the help with strains.

Correspondence should be addressed to Anindya Ghosh-Roy at anindya@ nbrc.ac.in or Sandeep Kumar at sandyjan87@gmail.com.

https://doi.org/10.1523/ENEURO.0414-20.2021

Copyright @ 2021 Kumar et al.

This is an open-access article distributed under the terms of the Creative Commons Attribution 4.0 International license, which permits unrestricted use, distribution and reproduction in any medium provided that the original work is properly attributed.
Ghosh-Roy et al., 2010; Nix et al., 2011). Several molecular pathways controlling axon regrowth potential are identified using mechanosensory and motor neurons as model systems (Byrne and Hammarlund, 2017; Hisamoto and Matsumoto, 2017). The two posterior lateral microtubule (PLM) neurons and the DA9 motor neurons allowed correlating functional restoration with axon regrowth at the single neuron level (Abay et al., 2017; Basu et al., 2017; Ding and Hammarlund, 2018). However, it has not been tested whether physical exercise would promote axon regeneration and functional recovery. In C. elegans, a single or multiple swimming sessions show exercise-like features (Laranjeiro et al., 2017, 2019). Swimming sessions extend neuromuscular and gut health span, enhance learning ability, and protects against neurodegeneration (Laranjeiro et al., 2019). Exercise in an electrotactic flow chamber ameliorates the age-related degeneration (Chuang et al., 2016).

In this study, we have investigated the role of swimming exercise in improving functional restoration after axotomy of the PLM neurons. We found that a single swim session of 90 min could improve functional restoration through axon regeneration process regardless of age. This exercise regimen can also improve the age-related decline in touch response function. To understand how swimming exercise promotes functional restoration, we imaged and correlated the anatomic pattern of regrowth to the recovery index of function. This revealed that exercise promotes regrowth, self-fusion events, and postregrowth functional recovery. Improvement in functional restoration on swimming depends on the function of the cellular energy sensor AMP kinase-2/AAK-2 in both neurons and muscle.

\section{Materials and Methods}

\section{C. elegans strains}

All the strains were grown and maintained at $20^{\circ} \mathrm{C}$ in nematode growth media (NGM) under standard conditions (Brenner, 1974). We used the following strains: Bristol N2, aak-2(ok524) X, mlk-1(ok2471) V, dlk-1(tm4024) I, ebp-1 (tm1357) $V$, and unc-54(r293) I. The extrachromosomal DNA-containing strains used were aak-2 (ok524); shrEx362 (Pmec-4::aak-2), aak-2(ok524); shrEx364 (Pmyo-3::aak-2), and aak-2(ok524); shrEX420 (Pdpy-7::aak-2). First, the extrachromosomal arrays were obtained in Pmec-7::GFP (muls32) background by injecting the rescue transgenes at $10 \mathrm{ng} / \mu \mathrm{l}$. Then the transgenes were introduced into the aak-2 mutant backgrounds by crossing. Homozygosity for all mutations was confirmed by either PCR or sequencing. All loss of function mutations are denoted as (0). We used the following transgenes: Pmec-7::GFP (muls32), Pmec-4:: GFP (zdls5) (Basu et al., 2017) and Pmec-4::mcherry::RAB3 (tbls227) (Sood et al., 2018).

\section{Age synchronization of worms}

Fifty gravid adults were transferred to fresh NGM plates seeded with OP50 for egg-laying and kept at $20^{\circ} \mathrm{C}$ for $2-3$ h. Worms were removed from the plates after they had laid eggs. The eggs were allowed to hatch and after $2 d$, 40-50 L4 worms were transferred to a fresh NGM plate 
containing $50 \mu \mathrm{M}$ 5-Fluoro deoxyuridine (FUDR; Sigma; catalog \#F0503; Basu et al., 2017). The worms at different life stages were used for experiments.

\section{Swimming exercise paradigm}

A single swimming session is considered as acute exercise paradigm in C. elegans (Laranjeiro et al., 2017, 2019). In our study, we have adopted mostly the single swimsession paradigm for exercise after axotomy of PLM neurons (Fig. 1A; Movie 1). Occasionally, the worms were subjected to multiple swim sessions (Fig. 2C). The worms were subjected to swimming in M9 buffer in 96-well plate for the duration ranging from 15 to $120 \mathrm{~min}$. Single animals were kept in wells containing $200 \mu$ l of M9 buffer (one worm per well). After the desired duration of swimming, the animals were recovered on NGM plate. In the control group, worms were kept in a plate without OP50 food for the same duration and then returned to plates containing food. We found that the ATP level is significantly dropped as reported before (Chaudhari and Kipreos, 2017) after a 90-min swim session and therefore we used this 90 -min session for most of the experiments.

\section{Measurement of ATP level after swim session}

To measure the change in the ATP level following swim exercise, we used an ATP bioluminescence assay kit CLSII (Roche Diagnostics, catalog \#11699695001; Palikaras and Tavernarakis, 2016). Briefly, 60 A3 worms from "non-swimming" as well as the "swimming" group were collected in $50 \mu \mathrm{l}$ of M9 buffer in a 1.5-ml tube. The samples were then frozen in liquid nitrogen. The frozen tubes were kept in boiling water for $15 \mathrm{~min}$. The samples were centrifuged at $14,800 \times g$ for $10 \mathrm{~min}$ at $4^{\circ} \mathrm{C}$. The supernatants were transferred to a fresh tube and diluted tenfold by adding water before measurement. The ATP levels were determined using Glomax luminometer (Promega). Before measurement, $100 \mu \mathrm{l}$ of sample or ATP standard was added to $100 \mu \mathrm{l}$ of luciferase in the well and incubated for $10 \mathrm{~s}$ at room temperature and then the luminescence was measured. The ATP level in the worm sample was derived from the ATP standard curve. Finally, the ATP levels were normalized with respect to the total protein $(\mathrm{mg})$ measured through BCA protein estimation method.

\section{Femtosecond laser, axotomy, and imaging}

For axotomy of PLM neurons, the animals were immobilized using $0.1-\mu \mathrm{m}$ polystyrene beads on a $5 \%$ agarose pad under a coverslip. For all the experiments, only one PLM axon corresponding to either left or right side of the animal was axotomized at a distance of 50-60 $\mu \mathrm{m}$ from the cell body, as described before (Basu et al., 2017). The side corresponding to the axotomized PLM neuron is called "cut side" and other called the "control side." Simultaneous two-photon imaging and axotomy was performed according to the previous published protocol (Basu et al., 2017). The PLM axon was imaged and axotomized with 920- and 720-nm lasers, respectively, under a $60 \times$ (Olympus) water-immersion objective of $1.1 \mathrm{NA}$ on a two-photon microscope (Basu et al., 2017). This system has two tunable (wavelength range 690-1040 nm), automated depression compensated femtosecond lasers from Spectra Physics (Mai Tai with Deepsee). The imaging was done with a 6-mm galvanometer scanning system and axotomy was performed with a 3-mm galvanometer system.

\section{Gentle touch assay}

Each worm was recovered after axotomy on NGM plate and then gentle touch assay was performed. Gentle Touch Response (Chalfie and Sulston, 1981; Chalfie et al., 1985; Basu et al., 2017) was assayed from both right and left sides of the worm. Following the touch assay in one side, the worm was flipped and kept for 20 min before touching the other side (Basu et al., 2017). Ten alternative anterior and posterior touches were given with the eyelash tip. The anterior touch was given to a forward-moving animal, which in response started moving backward. When a backward moving animal was given a posterior touch, it started moving forward in response. A positive response was denoted as 1 , and no response as 0 . Then the posterior touch response index (PTRI) was measured as the ratio of total number of positive response to total number of touch stimuli applied (Extended Data Fig. 1-1, Fig. 3B-C) as described before (Basu et al., 2017).

\section{Correlation of functional recovery with axon regeneration events at the level of a single worm}

At $3 \mathrm{~h}$ after axotomy, PTRls of both axotomized and control (uncut) side, were denoted as the PTRI postaxotomy. Each worm was labeled based on the side of axotomy and kept in single plate at $20^{\circ} \mathrm{C}$. After $24 \mathrm{~h}, \mathrm{PTRI}$ values from both the sides were measured and compared with the corresponding values at the postaxotomy stages. For a given side, the Recovery Index was obtained by the following formula: recovery index $=\mathrm{PTRl}_{24 \mathrm{~h}} / \mathrm{PTRl}_{3 \mathrm{~h}}$ (Extended Data Fig. 1-2). After the behavioral test at $24 \mathrm{~h}$, the regrowth pattern of the PLM axon was scored either using a Leica DM5000 fluorescent microscope at 40x magnification or a Spinning disk confocal microscope (Fig. 4D, Fig. 5A). Specifically, it was noted whether it was a fusion event (Fig. 5C) or non-fusion event (Fig. 5F). For scoring successful fusion events, we carefully evaluated whether the proximal end has just touched the distal counterpart or it has successfully joined and fused with the distal end (Fig. 5C). In case of casual touching, the distal end eventually undergo degeneration, and these events are referred as reconnection (Fig. 5C; Neumann and Hilliard, 2019).

\section{Imaging of axon regrowth events}

At $24 \mathrm{~h}$ postaxotomy, for imaging of the regeneration events, the animals were immobilized using $10 \mathrm{~mm}$ levamisole hydrochloride. Axonal regrowth was imaged using a Zeiss 864 Axio-Observer Z1 microscope equipped with Yokogawa CSU-XA1 spinning-disk confocal scan-head and a Photometrics Evolve EMCCD camera, the images were taken using $63 \times$ oil objective of $1.46 \mathrm{NA}$. The Pmec7::GFP labeled PLM axon was imaged with $30 \%$ input 
A

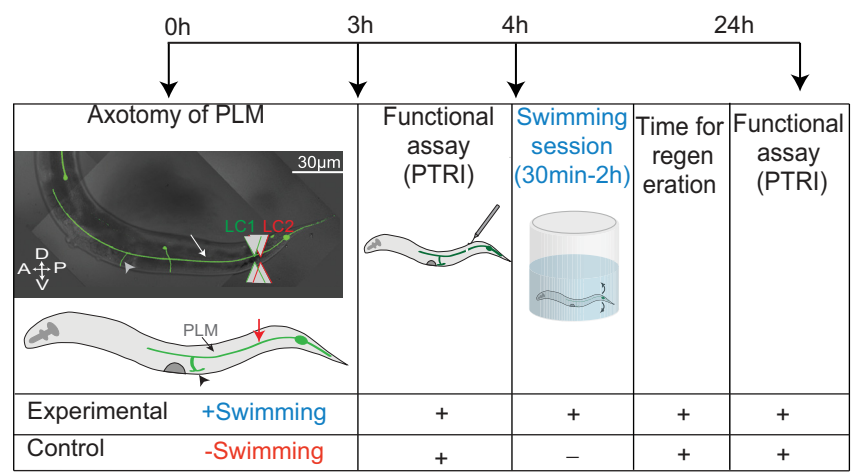

C

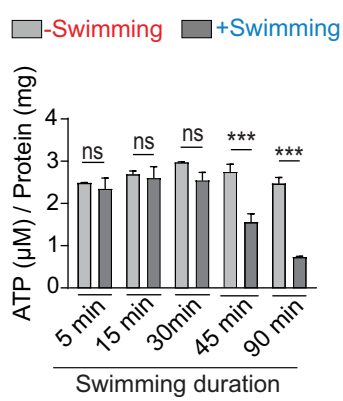

G
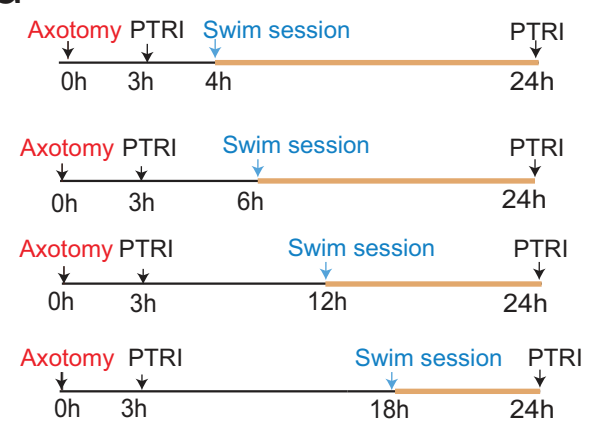

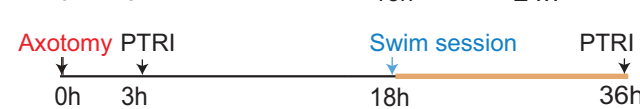

B

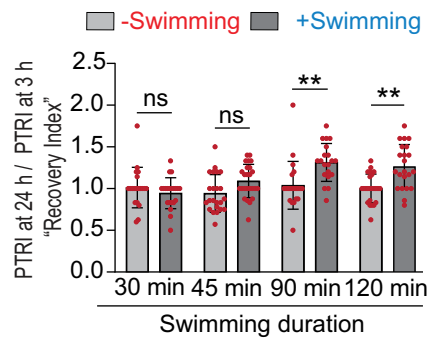

$E$

$\mathbf{F}$
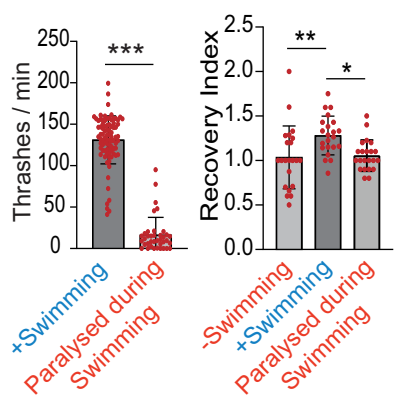

I

H
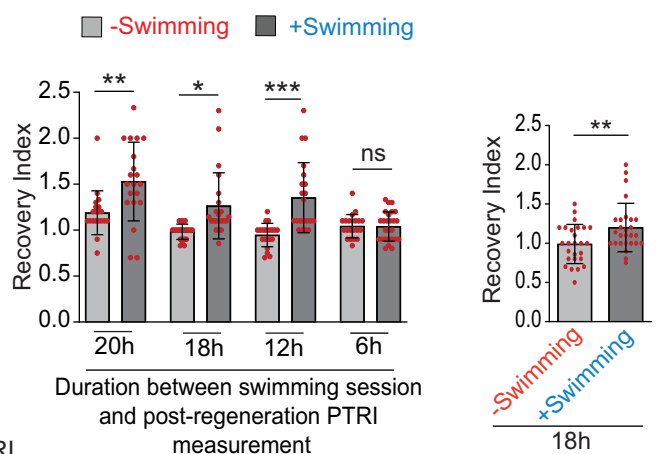

Figure 1. A single swim session after axotomy of PLM neurons enhances functional restoration in adult worm. $\boldsymbol{A}$, Experimental paradigm of swim session after the axotomy of a PLM neuron in A3 worms expressing Pmec-7::GFP (muls32) reporter. Following axotomy, the gentle touch response assay was performed to measure the reduction in PTRI (Extended Data Figure 1-1A). Then the animals were subjected to a swimming session for a duration ranging from $30 \mathrm{~min}$ to $2 \mathrm{~h}$ and recovered in NGM plate for further analysis. Another touch response assay was performed at $24 \mathrm{~h}$ postaxotomy to assess the functional recovery. Arrowhead denotes developmental synapses of PLM neurons. B, Quantification of the recovery index expressed as PTRI at $24 \mathrm{~h}$ postaxotomy/PTRI at $3 \mathrm{~h}$ postaxotomy for swim session of varying duration. $N=4-5$ independent replicates, $n=20-25$ number of animals tested. The raw data used for calculating recovery index values are given in Extended Data Figure 1-2. $\boldsymbol{C}$, The bar graph represents the ATP levels measured from the total extract prepared from 60 A3-stage animals after the swim session of varying duration, $N=4$ independent replicates. $\boldsymbol{D}$, Schematics for paralyzing the animals during the swim session. In order to prevent the worms from swimming in the well, they were treated with $5 \mathrm{~mm}$ levamisole for $15 \mathrm{~s}$ before the swimming session. The brief exposure to levamisole did not affect basal level PTRI (Extended Data Figure 1-1B). E. The bar graph represents the thrashing frequencies measured from the time-lapse imaging of the worms in the swimming well. $N=3-4, n=38-86$. $\boldsymbol{F}$, Recovery indices obtained at $24 \mathrm{~h}$ postaxotomy for control and paralyzed worms during the swim session, $N=3-4, n=22$. G, Scheme for determining the critical time required for seeing the beneficial effect of the swim session. In this experiment, the duration between swim session and postregeneration PTRI measurement was varied. $\boldsymbol{H}, \boldsymbol{I}$, Bar graphs showing the recovery indices measured according to the experimental paradigm described in $\mathbf{G}$ at $24 \mathrm{~h}$ postaxotomy and $36 \mathrm{~h}$ postaxotomy for $\boldsymbol{H}, \boldsymbol{I}$, respectively, $N=3-5, n=20-27$. Statistics, for $\boldsymbol{B}, \boldsymbol{C}, \boldsymbol{F}, \boldsymbol{H},{ }^{\star} p<0.05,{ }^{* *} p<0.01$, ${ }^{\star * \star} p<0.001$ ANOVA with Tukey's multiple comparison test. For $\boldsymbol{E}, \boldsymbol{I}$, ${ }^{\star *} p<0.01$, ${ }^{* * *} p<0.001$; unpaired $t$ test. Error bars represent SD; ns, not significant. 


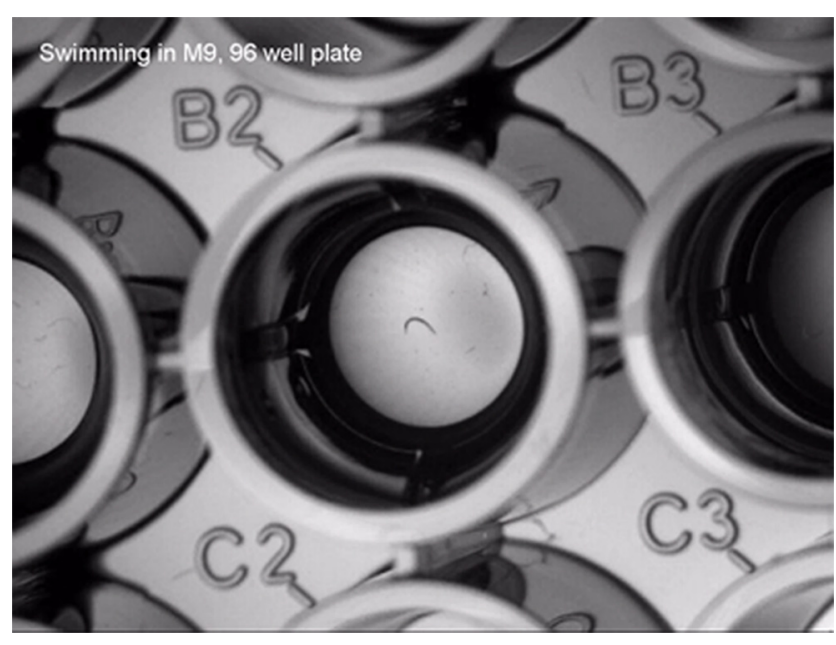

Movie 1. After the measurement of PTRI, worms were transferred to wells and allowed to swim for $90 \mathrm{~min}$. The videos were recorded at 15 frames/s (fps) and represented at $50 \mathrm{fps}$. [View online]

power of 480-nm laser. The images were acquired with the exposure time of $300 \mathrm{~ms}$ with the $70 \%$ of camera gain settings. The images were then exported as czi files and analyzed using ImageJ software. For regrowth length measurement, simple neurite tracer plugin of ImageJ was used. Pmec-4::mcherry::RAB-3 (tb/s227) reporter was used to image the formation synapse-like structures during axon regeneration. Imaging was done using a Nikon A1 plus (Nikon corporation) confocal microscope. PLM neurons expressing muls32 and tb/s227 reporter were simultaneously imaged after $24 \mathrm{~h}$ of axotomy. Imaging was done under a $60 \times$ oil objective $(\mathrm{NA}=1.4)$ at $1 \mu \mathrm{m}$ slice interval. Excitation power was 0.8 and 1 for 488- and 561$\mathrm{nm}$ laser, respectively. The PMT power for 488-nm channel was 100 and 104 for $561-n m$ channel with offset value of 20.

Each PLM neuron has a ventral branch (Fig. 1A, arrowhead), which makes synapse onto the postsynaptic interneuron (Chalfie and Sulston, 1981; Chalfie et al., 1985). This allowed us to set the dorsal-ventral axis of the worm (Fig. 5F) while analyzing the direction of axon regrowth. The axons that regrew up to $35-45 \mu \mathrm{m}$ in depth in ventral direction and extended along the ventral nerve cord were characterized as the "ventral targeting" event (Fig. 5FC). An accumulation of the presynaptic reporter mCherry:: RAB-3 along the ventral cord (Fig. 5FC, yellow arrowheads) was also noticed in these events. This accumulation pattern resembled the original chemical synapses of PLM neurons. When the distal end intensity was prominently less and showed beaded appearance, it was categorized as "distal degeneration" (Fig. 5FC) otherwise categorized as "distal intact" (Fig. 5Fa,b).

\section{Paralysis of the worms in swimming well}

At $3 \mathrm{~h}$ after axotomy, the animals were treated with 5 $\mathrm{mm}$ levamisole hydrochloride for $15 \mathrm{~s}$ to paralyze them. After this brief treatment with levamisole, they were placed in swimming wells. We found that this brief

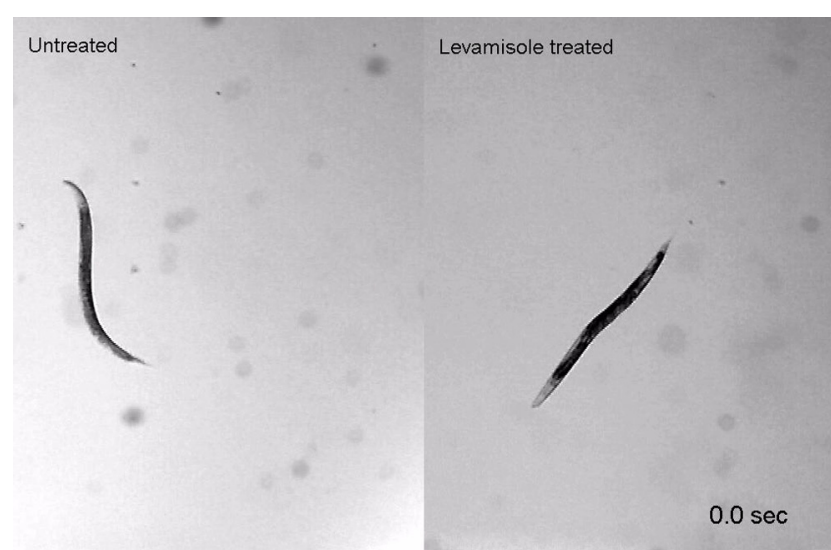

Movie 2. Swimming of control and paralyzed worms. In order to paralyze the worms, the worms were treated with $5 \mathrm{~mm}$ levamisole and transferred to the swimming well. Movies were acquired at 15 frames/s (fps) and represented at $50 \mathrm{fps}$. [View online]

treatment is sufficient to perturb the swimming (Movie 2; Fig. $1 E$ ) during the 90-min swim session. To verify whether this brief treatment of levamisole has any effect in the gentle touch response of the animal, we performed similar paralysis without performing axotomy and then measured the touch response after $24 \mathrm{~h}$. We found that the PTRI value was not affected by this brief treatment (Extended Data Fig. 1-1B). Therefore, we used this experimental design to test the effect of blocking the swimming on enhancement of functional regeneration (Fig. 1D-F).

\section{Measurement of thrashing frequency during swimming}

For the measurement of thrashing frequency, digital videos of worm movements were acquired during the swimming sessions, using a Leica MC 120 HD camera. The videos were recorded for $30 \mathrm{~s}$ at 15 frames/s using LAS V4.4 software in a Leica stereo microscope M165 FC at $1.25 \times$ magnification. The thrashing frequency were measured as body bends per second using wrMTrck plugin of ImageJ software (http://www.phage.dk/plugins/ wrmtrck.html). The change in the direction of bending at the mid-body was defined as one body bend (Truong et al., 2015). A mutant for muscle myosin unc-54 (Pulak and Anderson, 1988) showed drastically reduced thrashing frequency using this plugin. Thrashing frequency in unc-54 mutant was $18.27 \pm 13.04$ as opposed to $130.8 \pm 28.97$ for wild type. This suggests that our analysis is sensitive enough to show the difference in swimming ability because of various experimental conditions.

\section{Metformin treatment on paralyzed worms}

At $3 \mathrm{~h}$ postaxotomy, worms were first treated with $5 \mathrm{~mm}$ levamisole hydrochloride solution for $15 \mathrm{~s}$ to cause the muscle paralysis. The experimental swimming well had $50 \mathrm{~mm}$ Metformin hydrochloride (Sigma-Aldrich; catalog \#PHR1084). Therefore, the paralyzed worms were treated with Metformin during the 90-min swim session. Concentration of Metformin were chosen to activate AMPK/AAK-2 based on the previous report in C. elegans (Chen et al., 2017a). 


\section{Molecular biology and transgenes}

For touch neuron, muscle and epidermal-specific expression of aak-2, first a gateway (Thermo Fisher Scientific) entry clone of aak-2 [pCR8::aak-2 (pNBRGWY115)] was constructed by PCR with the primers $5^{\prime}$-ATGTITTCTCAT CAAGATCGAGA- ${ }^{\prime}$ and $5^{\prime}$-TCTCGATCTTGATGAGAAAAC AT- $3^{\prime}$. Then the entry clone was recombined with pCZGY553 (Pmec-4 destination vector), pCZGWY925 (Pmyo-3 destination vector) and pCZGWY44 (Pdpy-7 destination vector) to generate Pmec-4::aak-2 (pNBRGWY116), Pmyo-3::aak2 (pNBRGWY117) and Pdpy-7::aak-2 (pNBRGWY149), respectively.

\section{Statistics}

All the statistical analyses were performed using GraphPad Prism software version 9.0.2. For two-way comparisons, an unpaired $t$ test with Welch's correction was used. The median values were compared with the Mann-Whitney $U$ test. Fisher's exact test was used for proportions. Three or more samples were compared with ANOVA (nonparametric) with a post hoc Tukey's multiple comparisons test. The sample numbers $(n)$ presented on each bar are the total sample value accumulated over the total number of biological replicates $(N)$ in a given experiment.

\section{Results}

\section{A single swim session after axotomy of PLM neurons promotes functional recovery}

Previous studies have indicated that physical exercise following nervous system injury promotes axon regeneration and functional recovery in various model systems (Doyle and Roberts, 2006; Asensio-Pinilla et al., 2009; Sachdeva et al., 2016; Kuwabara et al., 2018). To address whether physical exercise can enhance functional restoration in C. elegans, we designed a swimming exercise paradigm in conjunction with PLM axon regeneration (Fig. $1 A$; Movie 1). A single session of swimming in $C$. elegans mimics the features of mammalian exercise (Laranjeiro et al., 2017). As previously reported that there is a sharp drop in functional recovery of gentle touch behavior after the axotomy of PLM neuron at day-3 adult worms (A3; Basu et al., 2017; Extended Data Fig. 1-1A), we tested whether a swim session after axotomy would enhance recovery. We measured the PTRI at 3 and 24 h postaxotomy (Fig. 1A). The extent of functional recovery was represented as the normalized PTRI at $24 \mathrm{~h}$ with respect to that measured at $3 \mathrm{~h}$ postaxotomy, which we called "recovery index" (Fig. 1B; Extended Data Fig. 1-2). A recovery index value, higher than 1 is an indication of improvement of touch response over the time after axotomy. A swimming session of $90 \mathrm{~min}$ or above significantly enhanced the recovery index at $24 \mathrm{~h}$ as compared with the non-swimming control (Fig. 1B; Extended Data Fig. 1-2). It also correlated with the significant drop in ATP level after the swimming session measured fluorometrically from the worm lysate (Fig. 1C). After 90 min, the worms did episodic swimming rather than continuous swimming (Ghosh and Emmons, 2008). Therefore, we chose 90 -min exercise window for our additional experiments. To confirm that the swimming-induced improvement in functional recovery is not because of the stress in liquid environment, rather because of exercise, we paralyzed the worm during swimming using levamisole that causes muscle hypercontraction (Culetto et al., 2004; Fig. 1D,E). The change in swimming ability was measured as thrashing frequency (Buckingham and Sattelle, 2009; Fig. 1E). We observed that the recovery index was significantly reduced when swimming was perturbed (Movie 2; Fig. 1F). Levamisole treatment in the swimming well per se did not affect the touch response index measured after the withdrawal of levamisole (Extended Data Fig. 1-1B).

To determine the time duration required for seeing the benefit of swimming, we varied the time window between the swim session and evaluation of touch response (PTRI; Fig. 1G). Essentially, the swim session was gradually shifted toward the time of postregeneration PTRI measurement (Fig. 1G). When the window was lower than 12 $\mathrm{h}$, in this case $6 \mathrm{~h}$, the functional improvement was nonsignificant (Fig. 1H). But when we increased the window, by shifting the time of postregeneration PTRI measurement further by $18 \mathrm{~h}$, the functional improvement was significant (Fig. 1/). Therefore, a critical time of $12 \mathrm{~h}$ is needed for proper manifestation of positive effect of swimming in regeneration.

\section{Multiple swim sessions are needed in older ages for the improvement in functional restoration}

Next, we expanded our single swim-session paradigm across various ages and found that this exercise regimen can improve the recovery index significantly when axotomy was performed at L4, A1, A3, and A4 stages (Fig. 2A; Extended Data Fig. 2-1). However, this improvement was non-significant at day 5 (A5; Fig. 2A; Extended Data Fig. $2-1)$. We noticed that the thrashing frequency during swimming was significantly reduced at A5 stage (Fig. 2B), which might have reduced the beneficial effect of swimming in functional restoration. We wanted to test whether multiple swimming sessions would be required at A5 stage for significant improvement in functional recovery. To test this hypothesis, we increased the number of swim sessions after the axotomy at $\mathrm{A} 5$ stage, one at $4 \mathrm{~h}$ and the other at $12 \mathrm{~h}$ postaxotomy (Fig. $2 \mathrm{C}$ ). Increasing the number of swim session raised the recovery index value significantly (Fig. 2D). Overall, our data suggest that swimmingrelated exercise promotes functional restoration regardless of age, although in older age, multiple exercise sessions are important.

\section{Swim exercise also prevents the age-related decline in touch neuron function}

It might be possible that swim exercise improves the touch neuron function in general, especially in older age when function is known to decline (Basu et al., 2017). As reported previously, we found that PTRI value is significantly dropped at A5 and subsequent life stages (Fig. 3B). A single swim session of $90 \mathrm{~min}, 1 \mathrm{~d}$ before the PTRI measurement (Fig. $3 A$ ) improved the PTRI value significantly 
A

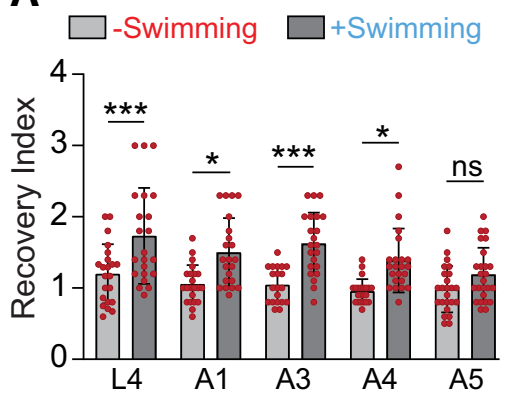

B
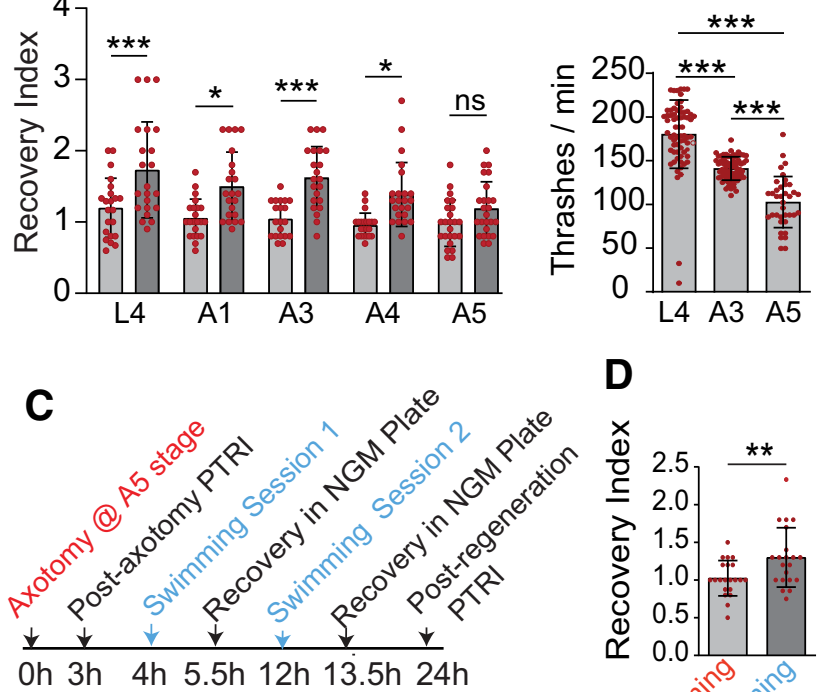

D

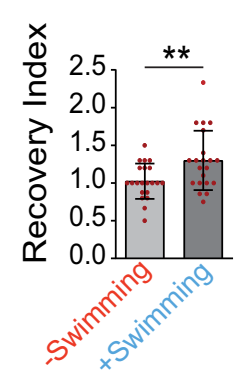

Figure 2. Multiple swimming-sessions after axotomy are critical for functional recovery in older ages. $\boldsymbol{A}$, The scatter plot with bars showing the effect of swimming exercise on functional recovery in L4 to A5 stage. The experiment was done in the transgenic background Pmec-7::GFP (muls32), $N=4-5$ independent replicates, $n=20-25$ number of animals tested. The raw data used for calculating recovery index values are given in Extended Data Figure 2-1. B, Thrashing frequency of worms during the swim session at different life stages $N=3, n=37-78$. C, Scheme for determining the effect of multiple swim sessions on functional recovery at A5 stage. Worms were allowed to swim for two sessions of $90 \mathrm{~min}$ each. $\boldsymbol{D}$, Recovery Indices in worms at $24 \mathrm{~h}$ postaxotomy, which underwent multiple swimming sessions after axotomy at A5 stage as shown in C-panel, $N=3, \quad n=21$. Statistics, for $\boldsymbol{A}, \boldsymbol{B},{ }^{*} p<0.05,{ }^{* *} p<0.01$, ${ }^{* * *} p<0.001$, ANOVA with Tukey's multiple comparison test. For $\boldsymbol{D},{ }^{\star *} p<0.01$, unpaired $t$ test. Error bars represent SD; ns, not significant.

at $A 5$ and $A 6$ stages (Fig. 3B,C). However, in A8 stage, the improvement in PTRI value because of single session swimming exercise was poor (Fig. 3B,C). No improvement at A8 stage was also noticed in the zdls5 (Pmec-4::GFP) transgenic reporter background (Fig. 3C). We asked whether a longer gap between swim session and functional assessment would be sufficient to enhance the PTRI value significantly at A8 stage. However, no further improvement in PTRI value was noticed when the exercise session was anticipated by $1 \mathrm{~d}$ (Fig. 3C). To test whether multiple swimming sessions across multiple days are helpful, we subjected the animals to four swimming sessions starting from A1 with 1-d interval between two sessions and then measured touch response at A8 stage (Fig. 3D). Multiple swimming sessions significantly elevated the PTRI value at $A 8$ stage as compared with that obtained from non-swimming control (Fig. $3 D, E$ ). Our data highlight that exercise improves both age-dependent decline in postaxotomy functional recovery, as well as prevents the age-related decline in touch neuron function. This raises the possibility that the exercise might only improve the neuronal function. Therefore, it needs to be resolved whether our exercise regimen would also enhance axon regrowth potential after axotomy.

\section{Swim session-mediated improvement in postaxotomy functional restoration involves initiation of axonal regeneration}

Physical exercise mediated improvement in functional recovery after neuronal injury often involves remodeling of spared neuronal circuits (van den Brand et al., 2015). Therefore, we wanted to test whether the benefit of swimming is because of compensatory mechanisms or regrowth of the axotomized PLM neuron. The p38-MAPK pathway involving DLK-1/MLK-1 is required in the early stages of axon regeneration in a cell-autonomous manner (Fig. 4A; Hammarlund et al., 2009; Yan et al., 2009; Ghosh-Roy et al., 2010). In the absence of this signaling cascade, injured axon cannot initiate the growth cone formation after axotomy. Therefore, the mutants affecting the DLK-1 cascade serve as a tool to block the regeneration from the injured proximal stump after axotomy (Fig. 4D, red arrows). A swim session neither in dlk-1(0) nor in $m / k-1(0)$ could promote functional restoration in A3 (Fig. 4B). Similar observation was made in dlk-1(0), when the experiment was conducted at L4 stage (Fig. 4C). Similarly, an upregulation of microtubule dynamics through microtubule plus-end binding protein-1 EBP-1 is critical for the efficient axon regrowth (Chen et al., 2011; Ghosh-Roy et al., 2012). We found that, the benefit of exercise was not seen in the absence of ebp-1 (Fig. 4B,C). Consistently, swimming could not promote the axon regrowth from the cut stump in these mutants (Fig. $4 D, E$, red arrows). To rule out the possibility of reduced swimming ability in these mutants, we analyzed the movies of their swimming. We found that thrashing frequency in these mutants are comparable to that in wild-type control (Fig. 4F).

These results confirm that exercise mediated increase in functional recovery involves regrowth from the injured proximal stump of the PLM neuron and subsequent rewiring into the functional circuit.

\section{Swim session promotes axon regrowth and postregrowth functional recovery}

Although swimming induced improvement in functional restoration involves initiation of axon regrowth after axotomy, it is not clear whether this exercise paradigm would enhance anatomic features of axon regrowth or it could simply enhance the functional aspect after axon regrows. The fact that swimming can enhance touch sensation behavior in older age, leaves the second possibility open. To test this, we performed confocal imaging of the regrowth events after assaying for the functional restoration (Fig. $5 A$ ), and correlated the behavioral recovery with the anatomic patterns of regeneration. We found that swimming exercise in A3 worms accelerated the initiation of the regrowth as revealed by the increased number of filopodia- 
A

$$
\begin{aligned}
& \text {-Swimming/ } \\
& \text { +Swimming } \\
& \text { PTRI } \\
& \text { measurement }
\end{aligned}
$$

B

muls32 [Pmec-7::GFP] -Swimming $\square$ +Swimming

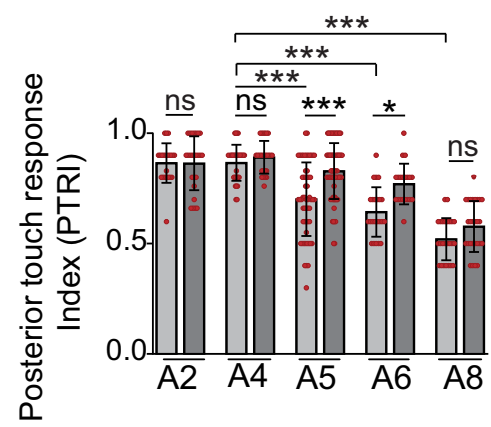

D zdls5[Pmec-4::GFP]

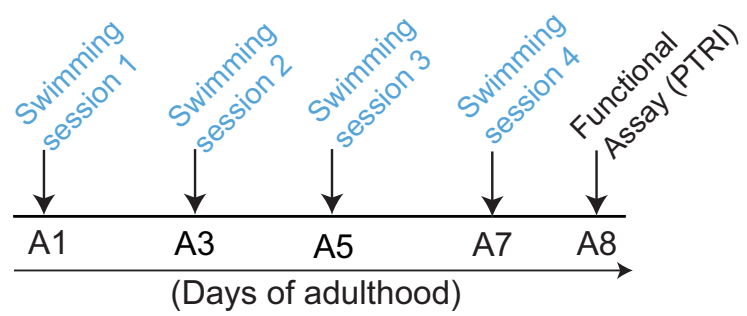

Days of Adulthood

\begin{tabular}{ccccc}
\hline A1 & A3 & A4 & A5 & A7 \\
\hline & $\vdots$ & & & $\vdots$ \\
\hdashline & $\vdots$ & $\vdots$ & $\vdots$ & $\vdots$ \\
A2 & A4 & A5 & A6 & A8
\end{tabular}

C

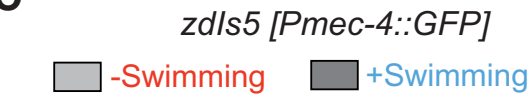

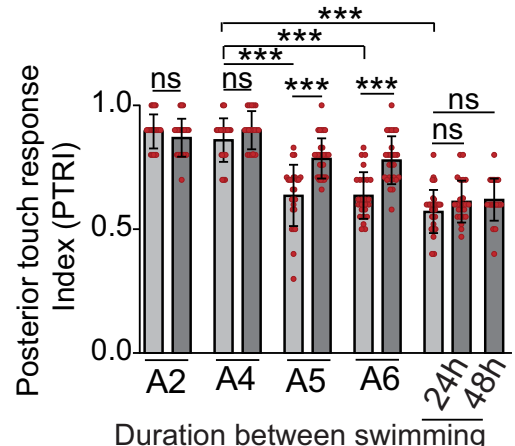
and functional assessment

E

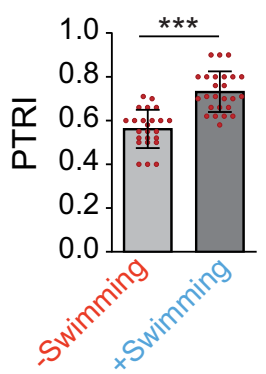

Figure 3. Swimming exercise prevents age-related decline in touch neuron function. $\boldsymbol{A}$, A paradigm to study the effect of swimming exercise of 90-min duration on age-dependent decline of posterior gentle touch response. Touch response assay was performed at $24 \mathrm{~h}$ postswimming session. $\boldsymbol{B}, \boldsymbol{C}$, Bar plots show the PTRls measured at $24 \mathrm{~h}$ postswimming session at various life stages in two different reporter backgrounds, Pmec-7::GFP (muls32; B) and Pmec-4::GFP (zdls5; C). In zdls5 background, an additional single swim session was performed at $48 \mathrm{~h}$ before PTRI measurement at A8 stage; $N=3-5$ independent replicates, $n=20-72$ number of worms tested. $\boldsymbol{D}$, Scheme for multiple swimming sessions to enhance touch response at A8 stage. $\boldsymbol{E}$, The effect of multiple swim sessions on PTRI values at A8 stage, $N=3, n=24-25$. Statistics, For $\boldsymbol{B}, \boldsymbol{C},{ }^{*} p<0.05,{ }^{* *} p<0.01,{ }^{* * *} p<0.001$ ANOVA with Tukey's multiple comparison test. For $\boldsymbol{E},{ }^{* \star \star} p<0.001$, unpaired $t$ test. Error bars represent SD; ns, not significant.

like extension at the cut stump at $6 \mathrm{~h}$ postaxotomy (Fig. 5B, arrowheads). The median value for the number of filopodia is increased from 1 to 2 because of the exercise session $\left({ }^{*} p=0.01\right.$, Mann-Whitney $U$ test). At $24 \mathrm{~h}$ postaxotomy, there was an enhancement in axonal regrowth as compared with the control condition (Fig. 5B). Regrowth value in the swimming group becomes $114.0 \pm 63.81 \mu \mathrm{m}$ as compared with the value $83.46 \pm 41.62 \mu \mathrm{m}$ obtained in non-swimming group ( ${ }^{*} p=0.04$, unpaired $t$ test). We also noticed that the percentage of self-fusion events with respect to the reconnection events (Ghosh-Roy et al., 2010; Neumann et al., 2011, 2015; Basu et al., 2017; Neumann and Hilliard, 2019) between proximal and distal ends are significantly increased because of the swimming session (Fig. $5 C, D$ ).
We further investigated whether the improvement in functional restoration seen because of swim exercise correlates with the "fusion" or "non-fusion" events, or both the categories of regrowth. We found that there is a significant increase in the recovery index for both the fusion event and non-fusion events (Fig. 5E). Upon correlating the regrowth pattern of the non-fusion events, we found that the regrowing axon, which goes toward the ventral cord and shows an enrichment of the presynaptic reporter mCherry::RAB-3 at the ventral cord (Fig. 5FC, arrowheads) corresponds to successful recovery in the swimming group as the value of the recovery index of this class is $2.66+0.73$ (Fig. $5 G$ ). We called this category of as ventral targeting events. The recovery index corresponding to the ventral targeting events 
A

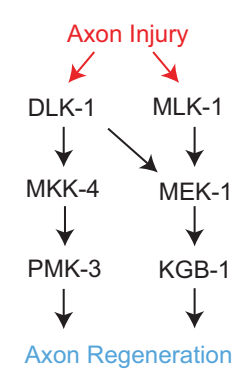

B

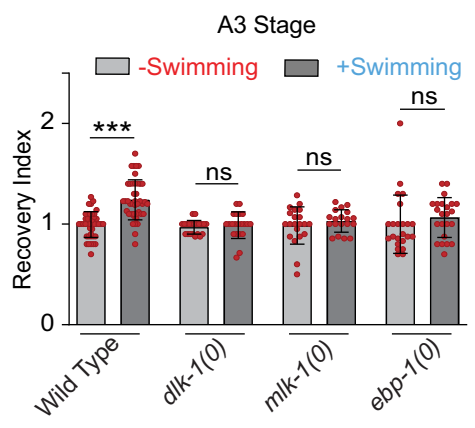

C

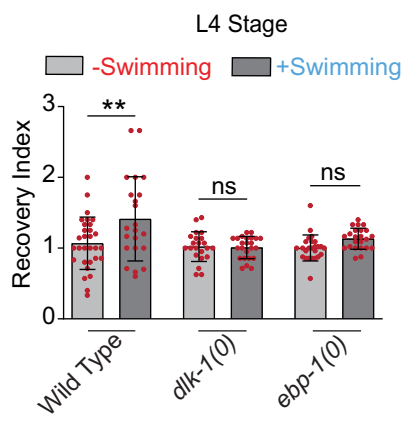

D

E

$\mathbf{F}$
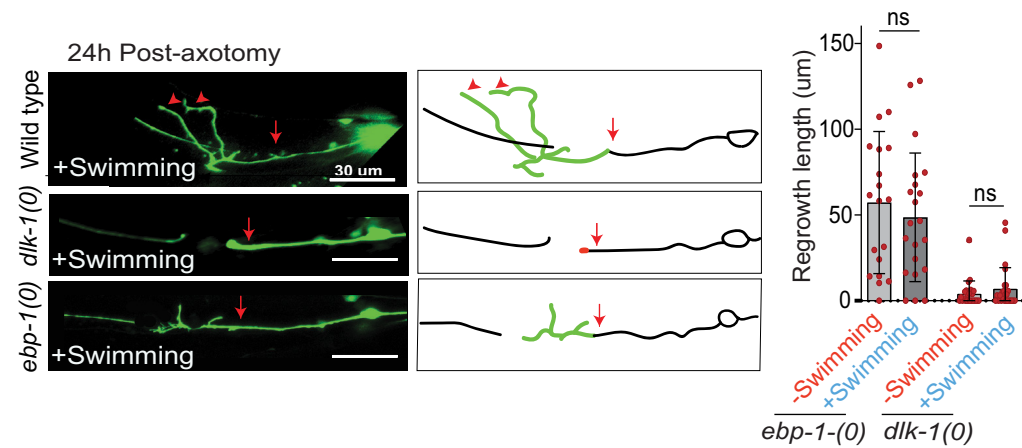

A3 stage

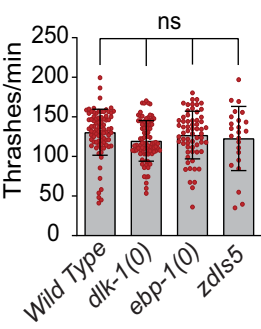

Figure 4. Swimming session-related improvement in functional recovery is dependent on axon regeneration. $\boldsymbol{A}$, Pathway diagram of two parallel p38-MAPK pathways involving DLK-1 and MLK-1 in axon regeneration. $\boldsymbol{B}$, The quantification showing the effect of swimming exercise on the recovery index measured at $24 \mathrm{~h}$ postaxotomy in the dlk-1(0), mlk-1(0), and ebp-1(0) at A3 stage. $N=3-4$ independent replicates, $n=19-42$ number of worms tested. C. The recovery indices measured at $24 \mathrm{~h}$ postaxotomy in the dlk-1(0) and ebp-1(0) at $L 4$ stage with and without swimming conditions $N=3-5, n=22-30$. $\boldsymbol{D}, \boldsymbol{E}$, Representative confocal images of PLM axons at $24 \mathrm{~h}$ postaxotomy in the wildtype control and mutants affecting axon regeneration pathways $(\boldsymbol{D})$ and axon regrowth values $(\boldsymbol{E})$ measured from the proximal cut tips (arrows in $\boldsymbol{D}), N=3-4, n=19-25$. Red arrowheads represent the filipodia-like structure at the regrowing tips, and arrows indicate the position of axotomy. $\boldsymbol{F}$, Thrashing frequencies in the mutants at A3 stage $N=3-4, n=24-85$. Statistics, for $\boldsymbol{B}, \boldsymbol{C}, \boldsymbol{E}, \boldsymbol{F},{ }^{* *} p<0.01,{ }^{* \star *} p<0.001$; ANOVA with Tukey's multiple comparison test. Error bars represent SD; ns, not significant.

was significantly higher as compared with the index obtained in the other classes such as "straight regrowth" (Fig. $5 F a)$ and "multibranch regrowth" (Fig. 5Fb). The percentage of ventral targeting events got increased on swim exercise (Fig. $5 H$ ). Since the distal end often persisted after injury (Fig. 5Fa,b), we asked whether the "intact distal end" could be contributing to the functional recovery. However, the recovery indices in the distal intact and "distal degenerated" categories were comparable in both swimming and non-swimming groups (Fig. 5/). Therefore, the enhancement of functional restoration because of swim exercise corresponds to successful rewiring process.

\section{Exercise-mediated improvement in touch neuron function requires metabolic energy sensor AAK-2}

During exercise, there is a consumption of energy in the form of ATP, which results in an increase in an AMP:ATP ratio (Chen et al., 2003). The increase in this ratio is sensed by the metabolic energy sensor kinase known as AMPK (Hardie, 2011). The physical exercise in mammalian system leads to activation of AMPK (Chen et al., 2003; Gibala et al., 2009). As in mammals, the two catalytic subunits of AMPK in C. elegans are encoded by two genes aak-1 and aak-2 (Apfeld et al., 2004). We hypothesized that the decrease in ATP levels after the 90min swimming session might be sensed by AAK-2 in neurons or muscle to regulate regeneration. To test this possibility, we gave the aak-2 mutant a 90-min swim session at various life stages starting from $A 1$ to $A 7$ and measured the posterior touch response (PTRI) after $24 \mathrm{~h}$ (Fig. 6A). We found that there is drop in touch response index value in aak-2 mutant starting from A5 stage similar to the wild type (Fig. 6B). However, the swim session could not elevate the PTRI in any of the life stages (Fig. $6 B$ ). We wondered, whether the abrogation of the exercise-induced elevation in PTRI value in aak-2(0) is due the reduced swimming ability of the mutant. However, the thrashing frequency in this mutant at $L 4, A 3$, and $A 5$ stages were comparable to the same values in wild-type control (Fig. $6 C)$. This indicated that AAK-2 might be specifically required for the exercise-induced changes in neuronal regeneration and function (Fig. 6D). To understand the tissue-specific requirement of aak-2 in this phenomenon, we have expressed aak-2 in muscle, epidermal cells and touch neuron exclusively. We found that both touch neuron and muscle-specific expression of aak-2 can rescue the swim session-induced phenomenon significantly (Fig. 6E). However, no rescue was observed when aak-2 was expressed in epithelial cells situated just next to the PLM axon 
A

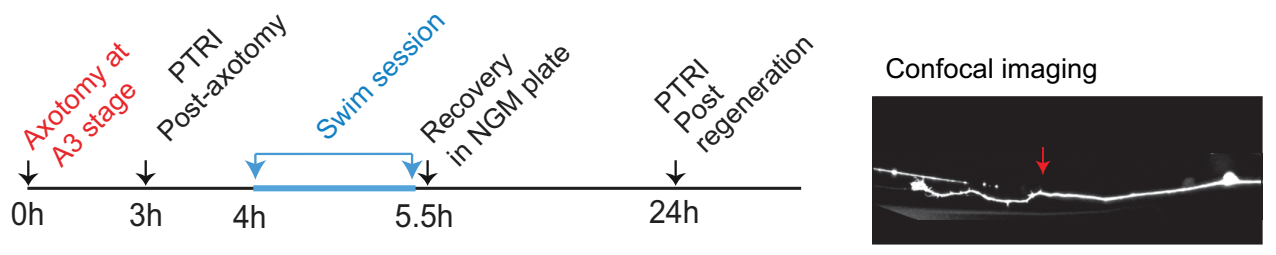

B

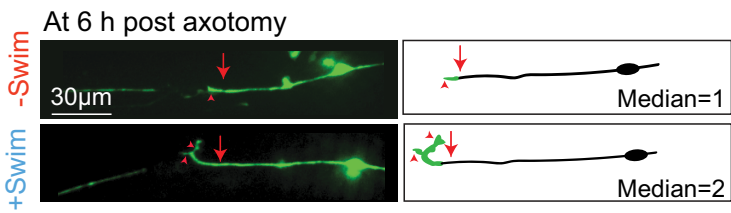

C

Fusion / Reconnection events

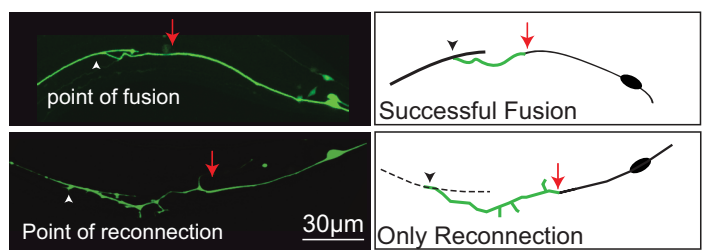

F Types of 'non-fusion' events

mCherry::RAB-3
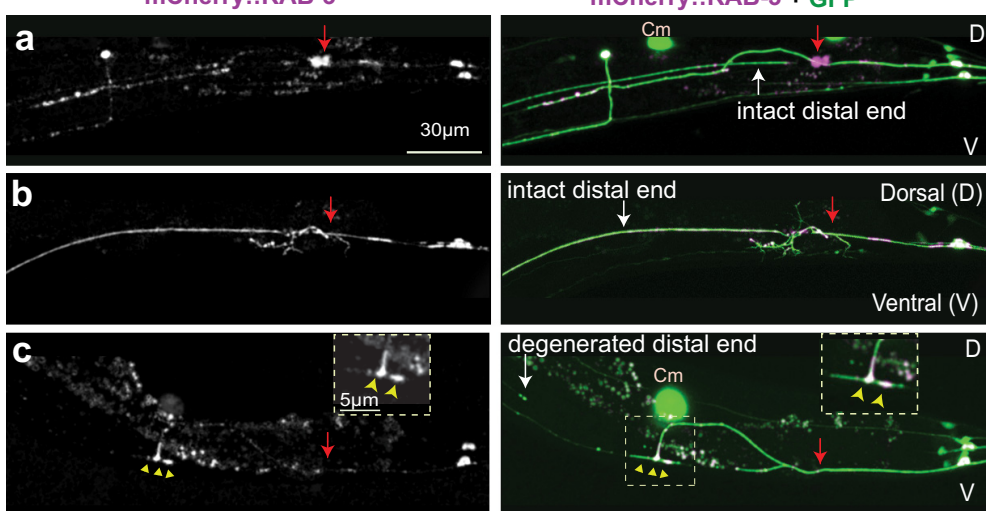

At $24 \mathrm{~h}$ post axotomy

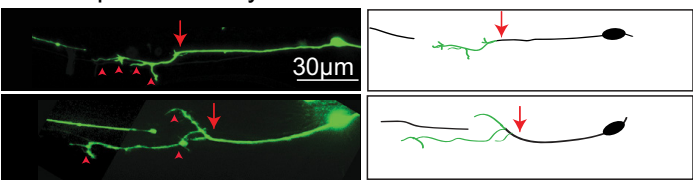

D

$\square$ Regrowth $\square$ Fusion/Reconnection

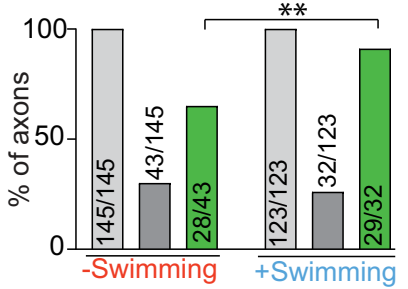

E $\square$-Swimming

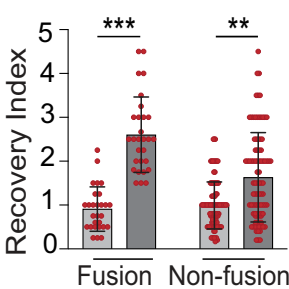

G

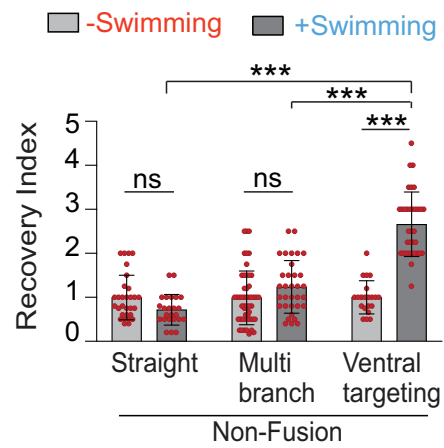

H $\square$-swimming +Swimming

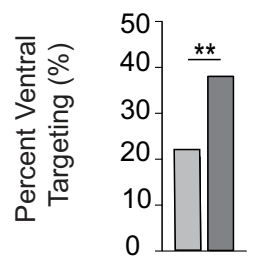

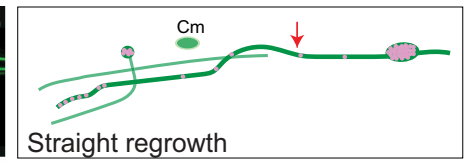

Straight regrowth

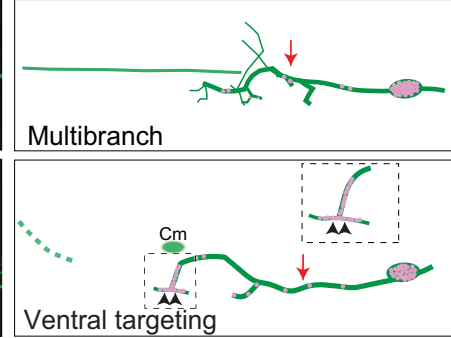

I $\square$ distal intact

distal degeneration

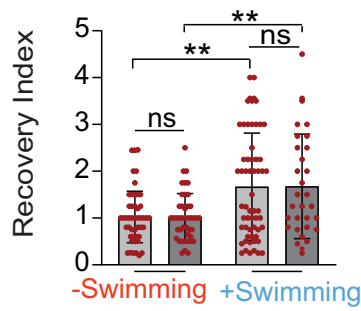

Figure 5. Swimming exercise promotes both axon regrowth and the functional recovery. $\boldsymbol{A}$, Experimental design to correlate functional recovery with the anatomic features of axon regrowth in worms expressing Pmec-7::GFP (muls32) at A3 stage. The confocal imaging was done after the measurement of PTRI values at the $24 \mathrm{~h}$ postaxotomy. $\boldsymbol{B}$, Representative images and illustration of PLM axons from control and swimming group at 6 and $24 \mathrm{~h}$ postaxotomy. Arrows and arrowheads indicate the position of axotomy and filopodia-like structures, respectively. C, Confocal images of fusion and "reconnection" events. Arrows indicate the position of axotomy. $\boldsymbol{D}$, Bar graph representing the $\%$ of different types of regeneration events in swimming and non-swimming groups, $N=3-5$ 
continued

independent replicates, $n=123-145$ neurons. $\boldsymbol{E}$, Bar graph with scatter plots presenting changes in the recovery index corresponding to the fusion and non-fusion events because of swim session. $N=3-5, n=52-91$ worms. $\boldsymbol{F}$, Confocal images representing different types of non-fusion regeneration events. The worms are expressing the presynaptic marker Pmec-4::mCherry-RAB3 (tb/s227) and Pmec-7::GFP (muls32). Yellow dashed box showing the enrichment of RAB-3 at the ventral cord region in the ventral targeting event. Arrowheads indicate enriched RAB-3 puncta. Red arrows indicate the position of axotomy; Cm denotes coelomocyte cell expressing the co-injection marker. G, Bar graph with scatter plots showing the changes in the recovery index because of swim exercise in different classes of non-fusion events, $N=3-5, n=21-39$. $\boldsymbol{H}$, The change in the \% of ventral targeting events because of swimming exercise. I, A comparison of recovery indices between the distal axon intact and distal axon degenerated events with respect to swimming exercise, $N=3-5, n=31-60$. Statistics, for $\boldsymbol{E}, \mathbf{G}, \boldsymbol{I},{ }^{* \star} p<0.01,{ }^{\star \star *} p<0.001$ ANOVA with Tukey's multiple comparison test. For $\boldsymbol{D}, \boldsymbol{H},{ }^{* \star} p<0.01 \chi^{2}$ test. Error bars represent SD; ns, not significant.

A

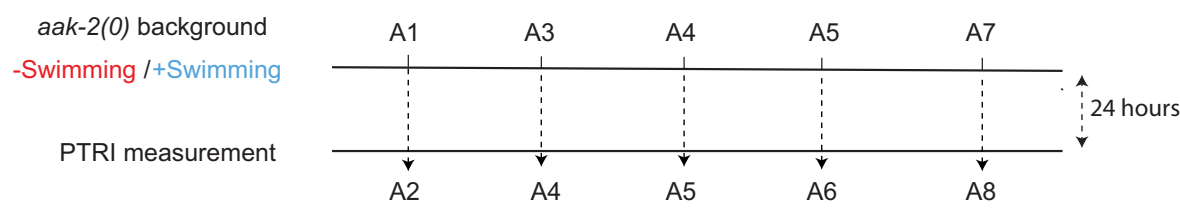

B

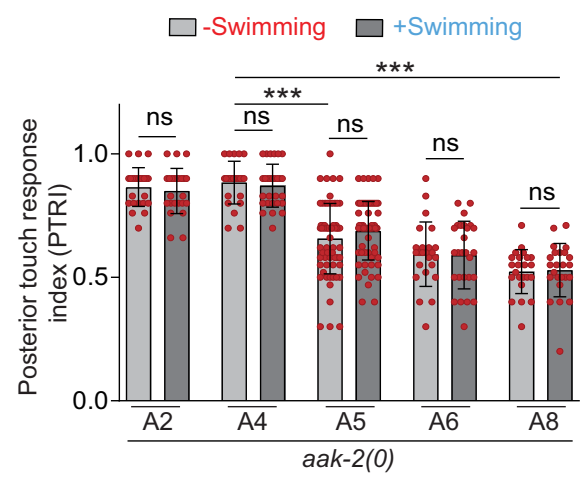

E

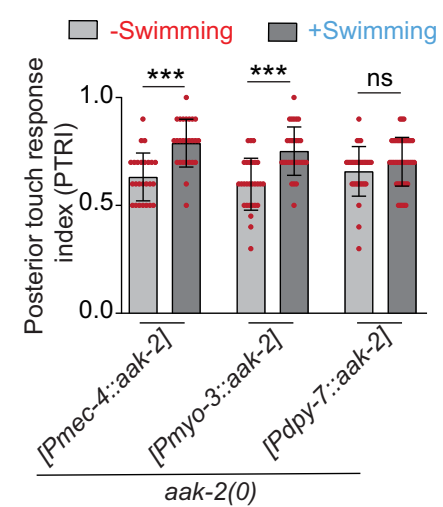

C

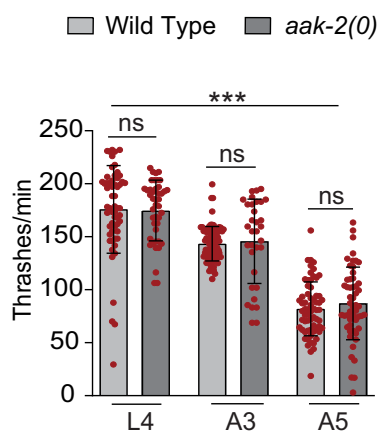

D

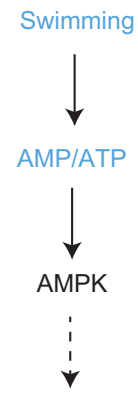

Functional Improvement
$\mathbf{F}$

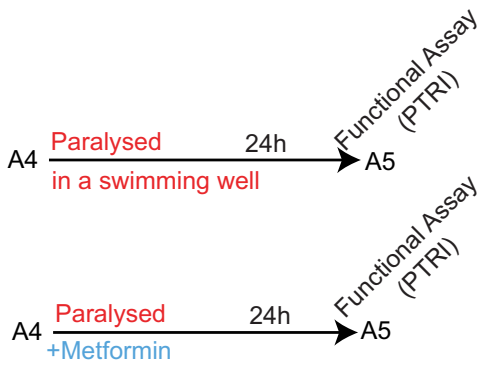

G

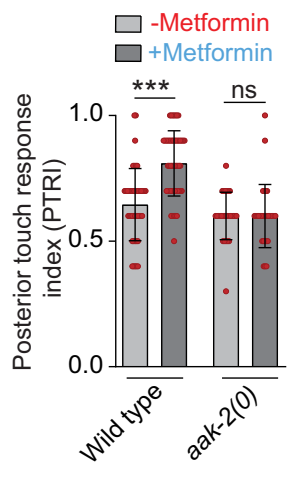

Figure 6. Swimming mediated improvement in touch response in older age is dependent on AAK-2. $\boldsymbol{A}$, Schematic to study the effect of single swimming session on age- related decline in touch neurons function in aak-2 mutant. Touch response assay was performed $24 \mathrm{~h}$ postswimming session. B. The PTRls measured at $24 \mathrm{~h}$ postswimming session in aak-2(0) at different life stages, $N=4-$ 6 independent replicates, $n=23-68$ number of animals. $\boldsymbol{C}$, The thrashing frequencies (body bends/min) in wild type and aak-2(0) at larval (L4) and adult stages, $N=3-4, n=33-90$. $\boldsymbol{D}$, A pathway diagram explaining how a swim session might enhance touch neuron function through AMPK. $\boldsymbol{E}$, The effect of swimming exercise on the decline in touch response at A5 stage in aak-2(0) expressing Pmec-4::aak-2 (shrEx362), Pmyo-3::aak-2 (shrEx364), and Pdpy-7::aak-2(shrEx420) transgenes, $N=3, n=25-41$. $\boldsymbol{F}$, Schematics of metformin treatment to the paralyzed worms in the swimming well to study the effect of AMPK activation in touch neuron function at A5 stage. The wild-type and aak-2(0) worms were pretreated with $5 \mathrm{~mm}$ levamisole for $15 \mathrm{~s}$ before the swimming session. $\mathbf{G}$, Changes in PTRI values at at $24 \mathrm{~h}$ posttreatment with $50 \mathrm{~mm}$ metformin as shown in $\boldsymbol{F}, N=5, n=28-51$. Statistics, for $\boldsymbol{B}$, $\boldsymbol{C}$, ${ }^{\star \star \star} p<0.001$; ANOVA with Tukey's multiple comparison test. For $\boldsymbol{E}, \mathbf{G}$, ${ }^{\star \star \star} p<0.001$; unpaired $t$ test. Error bars represent SD; ns, not significant. 

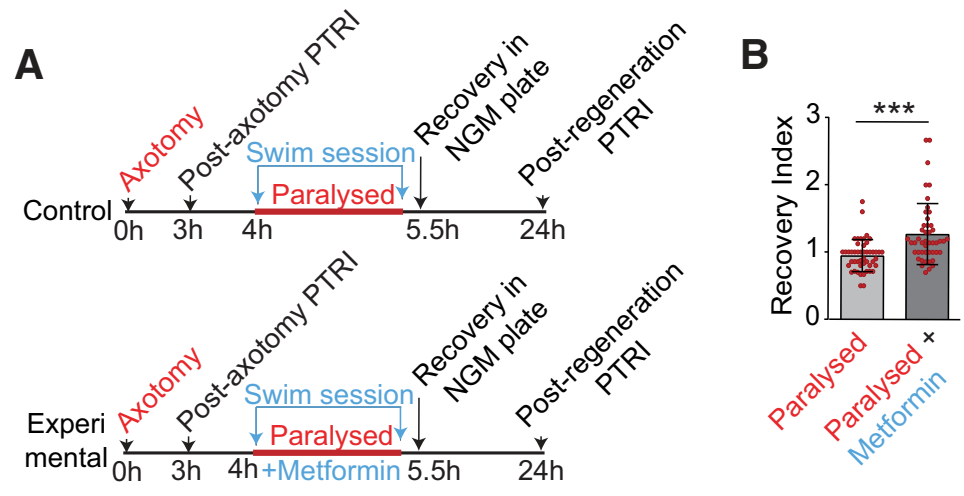

\section{C}
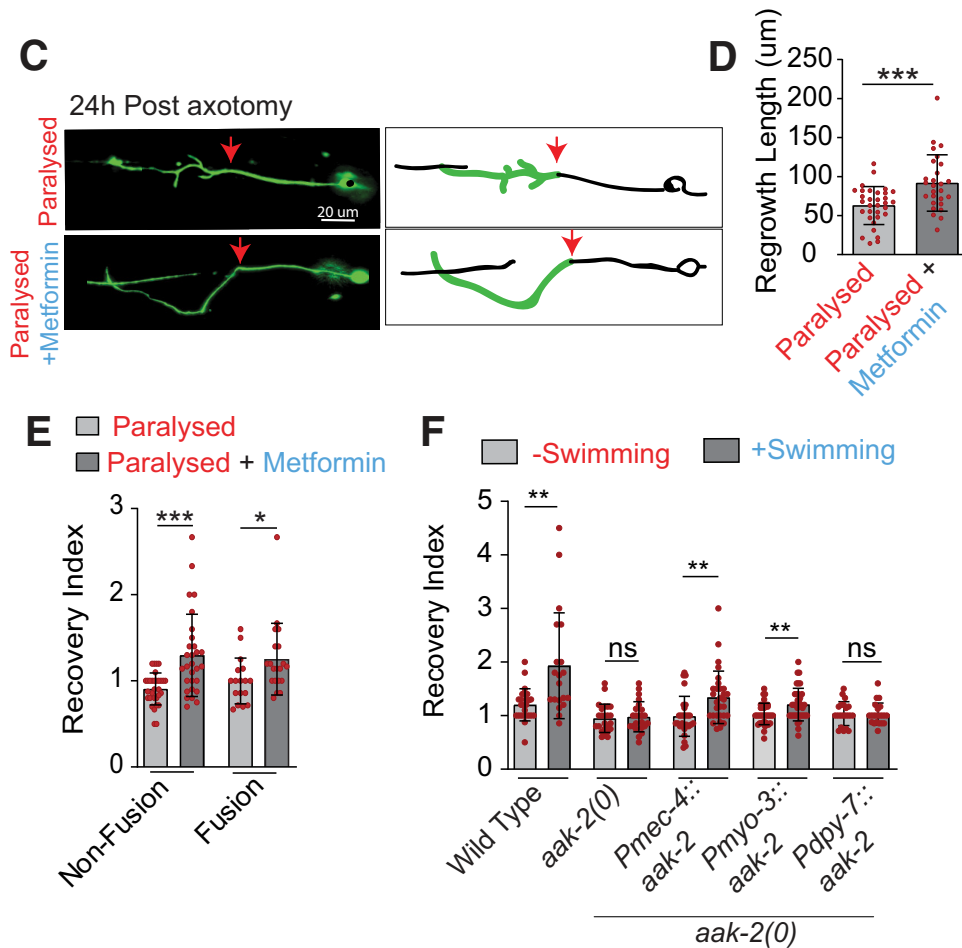

Figure 7. Swimming-mediated improvement in functional recovery after axon regeneration involves the energy sensor AAK-2. $\boldsymbol{A}$, Strategy for the AMPK activation during the swim session by treating with metformin. Wild-type worms were pretreated with 5 mM levamisole for $15 \mathrm{~s}$ before the swimming session to paralyze them. $50 \mathrm{~mm}$ metformin was applied to the paralyzed worms in the swimming well. $\boldsymbol{B}$, The effect of metformin treatment on recovery index at $24 \mathrm{~h}$ postaxotomy at $\mathrm{A} 3$ stage $N=5$ independent replicates, $n=47-48$ number of worms. $\boldsymbol{C}, \boldsymbol{D}$, Representative confocal images $(\boldsymbol{C})$ and quantification $(\boldsymbol{D})$ of PLM axon regrowth at $24 \mathrm{~h}$ postinjury in wild-type worms treated with or without metformin after axotomy at A3 stage. $N=5, n=27-32$. Arrows indicate the position of axotomy. $\boldsymbol{E}$, Bar graph represents the comparison of recovery index in control and metformin-treated worms at A3 stage, $N=3, n=48-49$. $\boldsymbol{F}$, The effect of touch neuron, muscle and epidermal cell-specific expression of aak-2 in aak-2 mutant on swimming induced enhancement in recovery index. The rescue transgenes Pmec-4::aak-2 (shrEx362), Pmyo-3::aak-2 (shrEx364), and Pdpy-7:: aak-2 (shrEx420) were used, $N=3, n=20-37$. The regrowth length and basal level of recovery index values in aak-2(0) mutant with or without the transgenes of aak-2 at L4 stage is presented in Extended Data Figure 7-1. Statistics, for, $\boldsymbol{B}, \boldsymbol{D}, \boldsymbol{E}, \boldsymbol{F},{ }^{*} p<0.05$, ${ }^{* *} p<0.01,{ }^{* \star *} p<0.001$ unpaired $t$ test. Error bars represent SD; ns, not significant.

(Fig. 6E). Next, we tested whether the AMPK activator metformin (Foretz et al., 2014; Rena et al., 2017) can mimic the benefits of swimming in touch neuron function (Fig. 6F). To address this question, we treated the worms with $50 \mathrm{~mm}$ metformin while they were kept paralyzed in the swimming well for $90 \mathrm{~min}$ (Fig. 6F). Metformin treatment was sufficient to enhance the PTRI value in A5 worms (Fig. 6G). This effect of metformin was absent in aak-2 mutant (Fig. 6G) indicating that effect of metformin in our assays is specific to AAK-2 function. These observations suggest that neuron-specific and muscle-specific activity of AAK-2 is important for swimexercise-induced enhancement of touch neuron function.

\section{Activation of the energy sensor AAK-2 after axotomy is sufficient to promote axon regrowth and functional restoration}

To address the role of AMPK/AAK-2 during swimming, we treated the 3-d-old (A3) worms at $3 \mathrm{~h}$ postaxotomy with $50 \mathrm{~mm}$ metformin only during the 90-min swim 


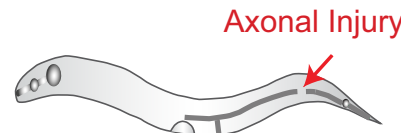

No swimming
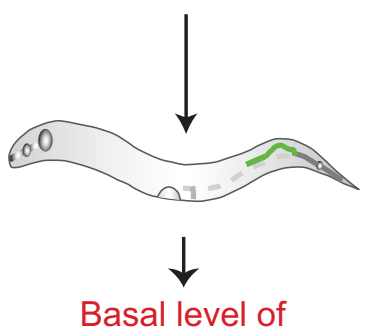

axon regeneration

and

Functional recovery
Axonal Injury

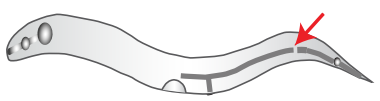

Swimming Exercise

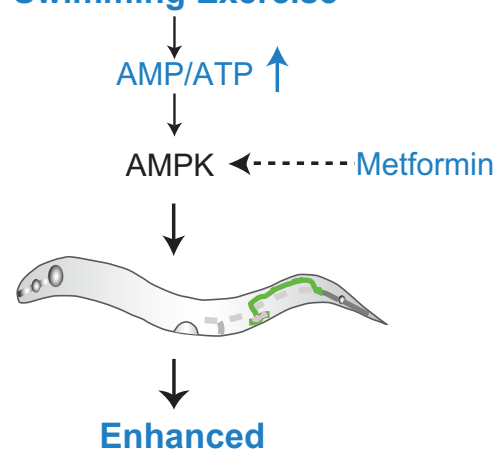

axon regeneration

and

\section{Functional recovery}

Figure 8. Proposed model illustrating how swimming exercise promotes axon regeneration. Exercise session after axonal injury leads to the consumption of cellular ATP resulting in activation of AMPK. An activated form of AMPK promotes axon regeneration and functional restoration.

session, while these animals were paralyzed with levamisole in the swimming well (Fig. 7A). Previously we have shown that when worms are paralyzed during the swim session, enhancement of functional restoration is blocked (Fig. 1F). We found that metformin treatment significantly enhanced the recovery index as compared with the nontreated control (Fig. 7B). Total regrowth from the proximal stump (Fig. 7C, red arrows) at $24 \mathrm{~h}$ postaxotomy was also increased significantly in the metformintreated group (Fig. 7C,D). The regrowth value becomes $91.77 \pm 36.19 \mu \mathrm{m}$ in metformin-treated group as compared with $62.87 \pm 24.36 \mu \mathrm{m}$ in untreated group. We noticed that there is an increase in the recovery index for both non-fusion-related and fusion-related events at $A 3$ stage (Fig. 7E). These observations suggest that AMPK activation is sufficient to mimic the effect of swimming exercise in axonal regeneration as well as functional recovery.

The aak-2 mutant shows reduced axon regrowth (Hubert et al., 2014; Extended Data Fig. 7-1A,B), which resulted in a loss of swim-exercise-mediated enhancement of postaxotomy recovery index at A3 stage (Fig. 7F). We found that the expression of aak-2 either in touch neurons or in muscle rescues the axon regrowth defect in aak-2 mutant at L4 stage (Extended Data Fig. 7-1A,B). However, the postinjury recovery index was only rescued significantly by the touch neuron-specific expression of aak-2 at L4 stage (Extended Data Fig. 7-1C). These transgenes also rescued the loss in benefit of swim session in functional recovery in aak-2 mutant at A3 stage (Fig. 7F). Expression of aak-2 in epithelial cells however did not rescue these phenotypes in aak-2 mutant (Fig. 7F; Extended Data Fig. 7-1A-C). These results indicate that AAK-2 acts both cell autonomously in neuron and noncell autonomously in muscle for transducing the effect of swim exercise in effective axon regeneration.

\section{Discussion}

In this study, using a well-established swimming paradigm we have studied the effect of physical exercise in functional recovery after the axonal injury of mechanosensory neuron. A swim session following axotomy promotes functional recovery regardless of age. This exercise regimen can also help overcome age-related decline in touch neuron function. By correlating anatomic features of axon regrowth with the behavioral index, we found that the swimming exercise enhances both postinjury axon regrowth as well as functional recovery. We further showed that the activity of the metabolic energy sensor AMPK in muscle as well as in injured neuron is critical in converting the energy spent during exercise into the positive effect in axon regeneration (Fig. 8).

In C. elegans, it is shown that a single swim session of 90 min presents exercise-like features such as muscle fatigue, a reduction of fat level in muscle, decrease in carbohydrate metabolism and enhanced mitochondrial oxidation (Laranjeiro et al., 2017). Many of these phenomena share commonalities with the physical exercise in vertebrate models (Thompson et al., 2001; Rivera-Brown and Frontera, 2012; Vina et al., 2012). In continuation to this finding, multiple and regular exercise sessions increase 
life-span, health quality, and protect against neurodegeneration in models of tauopathy, Alzheimer's disease, and Huntington's disease (Laranjeiro et al., 2019). It also increases overall learning ability as seen in other models and humans (Kobilo et al., 2014; Moon et al., 2016). These findings made it very relevant to address the role of physical exercise in promoting the repair of the injured neuronal circuitry using a worm model. We found that a 90-min swim session after the axotomy is sufficient to overcome age-related decline in axon regeneration and functional restoration. Intriguingly, this exercise paradigm is also sufficient to improve the functional recovery in early stages of life. Previous literature has demonstrated the benefit of physical exercise in peripheral nerve regeneration (Park and Höke, 2014; Gordon and English, 2016; Chen et al., 2017b). Since our behavioral assay is specific to single neuron responsible for touch sensation, we provided a direct evidence that enhanced axon regrowth because of physical exercise drives functional recovery.

An immediate effect of exercise is a reduction in the ratio of ATP/AMP (Chen et al., 2003), which is sensed by AMP Kinase (Hardie, 2011). Following the swimming session, we recorded a significant drop in ATP level. Therefore, we speculated a role of AMPK/AAK-2 in exercise-mediated improvement in touch neurons function. Consistent with this hypothesis, the loss of aak-2 significantly abolished the improvement in touch neurons regeneration and function because of swimming exercise. Conversely, activation of AMPK using metformin was sufficient to promote axon regeneration. Tissue-specific rescue experiments suggested that AAK-2 acts both in neuron and muscle for enhanced axon regeneration and functional recovery. Few studies indicated that activation of AMPK by metformin has positive effect after spinal cord injury (Zhang et al., 2017; Guo et al., 2018). Pharmacological activation of AMPK can promote muscle fiber regeneration in a mouse myopathy model (Peralta et al., 2016). The AMPK agonist AICAR enhances spatial memory in wild-type animals, but this improvement was lacking in muscle-specific mutant of AMPK pointing toward the link between muscles and nervous system (Kobilo et al., 2014).

The question is how activated AAK-2 could be enhancing axon regeneration. It has been observed that postexercise activation of AMPK leads to the ATP synthesis via various metabolic pathways (Winder and Hardie, 1996; Hutber et al., 1997; Hardie, 2004). It also leads to mitochondrial biogenesis through proliferator-activated receptor $\gamma$ coactivator-1 $\alpha$ (PGC-1 $\alpha$; Zong et al., 2002; Kukidome et al., 2006). After a neuronal injury, the growth cone formation and subsequent regrowth is driven by various enzymes and motors. This requires high level of energy (Bradke et al., 2012; Zhou et al., 2016). Therefore, AMPK-driven ATP production can boost up the initiation of axon regeneration process as seen in our analysis. Apart from restoring the cellular energy levels, AMPK also regulates autophagy which allows the cell to survive the metabolic stress (Zhao and Klionsky, 2011). Autophagy induction has been linked with the stabilization of microtubules and enhancement of axonal regeneration after neuronal injury ( $\mathrm{He}$ et al., 2016; Ko et al., 2020). Other possibility through which the AMPK can enhance axon regeneration is through DAF-16/FOXO1 activation. The activated AMPK can directly phosphorylate and regulate DAF-16 (Greer et al., 2007), which is known to regulate axon regeneration (Byrne et al., 2014). It would be an interesting direction to unravel how AMPK signaling couples muscle and neuron in neuronal regeneration.

\section{References}

Abay ZC, Wong MY, Teoh JS, Vijayaraghavan T, Hilliard MA, Neumann B (2017) Phosphatidylserine save-me signals drive functional recovery of severed axons in Caenorhabditis elegans. Proc Natl Acad Sci USA 114:E10196-E10205.

Apfeld J, O'Connor G, McDonagh T, DiStefano PS, Curtis R (2004) The AMP-activated protein kinase AAK-2 links energy levels and insulin-like signals to lifespan in C. elegans. Genes Dev 18:30043009.

Asensio-Pinilla E, Udina E, Jaramillo J, Navarro X (2009) Electrical stimulation combined with exercise increase axonal regeneration after peripheral nerve injury. Exp Neurol 219:258-265.

Basu A, Dey S, Puri D, Das Saha N, Sabharwal V, Thyagarajan P, Srivastava P, Koushika SP, Ghosh-Roy A (2017) let-7 miRNA controls CED-7 homotypic adhesion and EFF-1-mediated axonal selffusion to restore touch sensation following injury. Proc Natl Acad Sci USA 114:E10206-E10215.

Bradke F, Fawcett JW, Spira ME (2012) Assembly of a new growth cone after axotomy: the precursor to axon regeneration. Nat Rev Neurosci 13:183-193.

Brenner S (1974) The genetics of Caenorhabditis elegans. Genetics 77:71-94.

Brosius Lutz A, Barres BA (2014) Contrasting the glial response to axon injury in the central and peripheral nervous systems. Dev Cell 28:7-17.

Buckingham SD, Sattelle DB (2009) Fast, automated measurement of nematode swimming (thrashing) without morphometry. BMC Neurosci 10:84.

Byrne AB, Hammarlund M (2017) Axon regeneration in C. elegans: worming our way to mechanisms of axon regeneration. Exp Neurol 287:300-309.

Byrne AB, Walradt T, Gardner KE, Hubbert A, Reinke V, Hammarlund M (2014) Insulin/IGF1 signaling inhibits age-dependent axon regeneration. Neuron 81:561-573.

Capogrosso M, Milekovic T, Borton D, Wagner F, Moraud EM, Mignardot JB, Buse N, Gandar J, Barraud Q, Xing D, Rey E, Duis S, Jianzhong Y, Ko WKD, Li Q, Detemple P, Denison T, Micera S, Bezard E, Bloch J, et al. (2016) A brain-spine interface alleviating gait deficits after spinal cord injury in primates. Nature 539:284288.

Chalfie M, Sulston J (1981) Developmental genetics of the mechanosensory neurons of Caenorhabditis elegans. Dev Biol 82:358-370.

Chalfie M, Sulston JE, White JG, Southgate E, Thomson JN, Brenner $S$ (1985) The neural circuit for touch sensitivity in Caenorhabditis elegans. J Neurosci 5:956-964.

Chaudhari SN, Kipreos ET (2017) Increased mitochondrial fusion allows the survival of older animals in diverse $C$. elegans longevity pathways. Nat Commun 8:182.

Chen J, Ou Y, Li Y, Hu S, Shao LW, Liu Y (2017a) Metformin extends C. elegans lifespan through lysosomal pathway. Elife 6:e31268.

Chen YS, Way TD, Chen YH, Yao CH, Yang TY, Liao CF (2017b) Effects of swimming exercise on nerve regeneration in a rat sciatic nerve transection model. Biomedicine (Taipei) 7:3.

Chen L, Wang Z, Ghosh-Roy A, Hubert T, Yan D, O'Rourke S, Bowerman B, Wu Z, Jin Y, Chisholm AD (2011) Axon regeneration pathways identified by systematic genetic screening in $C$. elegans. Neuron 71:1043-1057.

Chen ZP, Stephens TJ, Murthy S, Canny BJ, Hargreaves M, Witters LA, Kemp BE, McConell GK (2003) Effect of exercise intensity on skeletal muscle AMPK signaling in humans. Diabetes 52:22052212. 
Chuang HS, Kuo WJ, Lee CL, Chu IH, Chen CS (2016) Exercise in an electrotactic flow chamber ameliorates age-related degeneration in Caenorhabditis elegans. Sci Rep 6:28064.

Culetto E, Baylis HA, Richmond JE, Jones AK, Fleming JT, Squire MD, Lewis JA, Sattelle DB (2004) The Caenorhabditis elegans unc63 gene encodes a levamisole-sensitive nicotinic acetylcholine receptor alpha subunit. J Biol Chem 279:42476-42483.

Ding C, Hammarlund M (2018) Aberrant information transfer interferes with functional axon regeneration. Elife 7:e38829.

Doyle LM, Roberts BL (2006) Exercise enhances axonal growth and functional recovery in the regenerating spinal cord. Neuroscience 141:321-327.

Foretz M, Guigas B, Bertrand L, Pollak M, Viollet B (2014) Metformin: from mechanisms of action to therapies. Cell Metab 20:953-966.

Formento E, Minassian K, Wagner F, Mignardot JB, Le GoffMignardot CG, Rowald A, Bloch J, Micera S, Capogrosso M, Courtine G (2018) Electrical spinal cord stimulation must preserve proprioception to enable locomotion in humans with spinal cord injury. Nat Neurosci 21:1728-1741.

Fu J, Wang H, Deng L, Li J (2016) Exercise training promotes functional recovery after spinal cord injury. Neural Plast 2016:4039580.

Geoffroy CG, Hilton BJ, Tetzlaff W, Zheng B (2016) Evidence for an age-dependent decline in axon regeneration in the adult mammalian central nervous system. Cell Rep 15:238-246.

Ghosh R, Emmons SW (2008) Episodic swimming behavior in the nematode C. elegans. J Exp Biol 211:3703-3711.

Ghosh-Roy A, Wu Z, Goncharov A, Jin Y, Chisholm AD (2010) Calcium and cyclic AMP promote axonal regeneration in Caenorhabditis elegans and require DLK-1 kinase. J Neurosci 30:3175-3183.

Ghosh-Roy A, Goncharov A, Jin Y, Chisholm AD (2012) Kinesin-13 and tubulin posttranslational modifications regulate microtubule growth in axon regeneration. Dev Cell 23:716-728.

Gibala MJ, McGee SL, Garnham AP, Howlett KF, Snow RJ, Hargreaves M (2009) Brief intense interval exercise activates AMPK and p38 MAPK signaling and increases the expression of PGC-1alpha in human skeletal muscle. J Appl Physiol (1985) 106:929-934.

Gordon T, English AW (2016) Strategies to promote peripheral nerve regeneration: electrical stimulation and/or exercise. Eur J Neurosci 43:336-350.

Greer EL, Dowlatshahi D, Banko MR, Villen J, Hoang K, Blanchard D, Gygi SP, Brunet A (2007) An AMPK-FOXO pathway mediates longevity induced by a novel method of dietary restriction in $C$. elegans. Curr Biol 17:1646-1656.

Guo Y, Wang F, Li H, Liang H, Li Y, Gao Z, He X (2018) Metformin protects against spinal cord injury by regulating autophagy via the mTOR signaling pathway. Neurochem Res 43:1111-1117.

Hammarlund M, Nix P, Hauth L, Jorgensen EM, Bastiani M (2009) Axon regeneration requires a conserved MAP kinase pathway. Science 323:802-806.

Hardie DG (2004) The AMP-activated protein kinase pathway-new players upstream and downstream. J Cell Sci 117:5479-5487.

Hardie DG (2011) AMP-activated protein kinase-an energy sensor that regulates all aspects of cell function. Genes Dev 25:18851908.

He M, Ding Y, Chu C, Tang J, Xiao Q, Luo Z-G (2016) Autophagy induction stabilizes microtubules and promotes axon regeneration after spinal cord injury. Proc Natl Acad Sci USA 113:11324-11329.

$\mathrm{He} Z$, Jin $Y$ (2016) Intrinsic control of axon regeneration. Neuron 90:437-451.

Hisamoto N, Matsumoto K (2017) Signal transduction cascades in axon regeneration: insights from $C$. elegans. Curr Opin Genet Dev 44:54-60.

Horowitz AM, Fan X, Bieri G, Smith LK, Sanchez-Diaz Cl, Schroer AB, Gontier G, Casaletto KB, Kramer JH, Williams KE, Villeda SA (2020) Blood factors transfer beneficial effects of exercise on neurogenesis and cognition to the aged brain. Science 369:167-173.
Hubert T, Wu Z, Chisholm AD, Jin Y (2014) S6 kinase inhibits intrinsic axon regeneration capacity via AMP kinase in Caenorhabditis elegans. J Neurosci 34:758-763.

Huebner EA, Strittmatter SM (2009) Axon regeneration in the peripheral and central nervous systems. Results Probl Cell Differ 48:339351.

Hutber CA, Hardie DG, Winder WW (1997) Electrical stimulation inactivates muscle acetyl-CoA carboxylase and increases AMP-activated protein kinase. Am J Physiol 272:E262-E266.

Johnson VE, Stewart W, Smith DH (2013) Axonal pathology in traumatic brain injury. Exp Neurol 246:35-43.

Karssemeijer EGA, Aaronson JA, Bossers WJ, Smits T, Olde Rikkert MGM, Kessels RPC (2017) Positive effects of combined cognitive and physical exercise training on cognitive function in older adults with mild cognitive impairment or dementia: a meta-analysis. Ageing Res Rev 40:75-83.

Ko SH, Apple EC, Liu Z, Chen L (2020) Age-dependent autophagy induction after injury promotes axon regeneration by limiting NOTCH. Autophagy 16:2052-2068.

Kobilo T, Guerrieri D, Zhang Y, Collica SC, Becker KG, van Praag H (2014) AMPK agonist AICAR improves cognition and motor coordination in young and aged mice. Learn Mem 21:119-126.

Kukidome D, Nishikawa T, Sonoda K, Imoto K, Fujisawa K, Yano M, Motoshima H, Taguchi T, Matsumura T, Araki E (2006) Activation of AMP-activated protein kinase reduces hyperglycemia-induced mitochondrial reactive oxygen species production and promotes mitochondrial biogenesis in human umbilical vein endothelial cells. Diabetes 55:120-127.

Kuwabara N, Nakamoto K, Shirose Y, Kiso N, Kokubun T, Murata K, Kanemura N (2018) Effect of exercise on the cell death and axonal regeneration in a rat model of peripheral nerve injury. Ann Phys Rehab Med 61:e261.

Laha B, Stafford BK, Huberman AD (2017) Regenerating optic pathways from the eye to the brain. Science 356:1031-1034.

Laranjeiro R, Harinath G, Burke D, Braeckman BP, Driscoll M (2017) Single swim sessions in $C$. elegans induce key features of mammalian exercise. BMC Biol 15:30.

Laranjeiro R, Harinath G, Hewitt JE, Hartman JH, Royal MA, Meyer JN, Vanapalli SA, Driscoll M (2019) Swim exercise in Caenorhabditis elegans extends neuromuscular and gut healthspan, enhances learning ability, and protects against neurodegeneration. Proc Natl Acad Sci USA 116:23829-23839.

Mahar M, Cavalli V (2018) Intrinsic mechanisms of neuronal axon regeneration. Nat Rev Neurosci 19:323-337.

Moon HY, Becke A, Berron D, Becker B, Sah N, Benoni G, Janke E, Lubejko ST, Greig NH, Mattison JA, Duzel E, van Praag H (2016) Running-induced systemic cathepsin B secretion is associated with memory function. Cell Metab 24:332-340.

Neumann B, Hilliard MA (2019) Axonal repair by fusion: pitfalls, consequences and solutions. FASEB J 33:13071-13074.

Neumann B, Nguyen KC, Hall DH, Ben-Yakar A, Hilliard MA (2011) Axonal regeneration proceeds through specific axonal fusion in transected C. elegans neurons. Dev Dyn 240:1365-1372.

Neumann B, Coakley S, Giordano-Santini R, Linton C, Lee ES, Nakagawa A, Xue D, Hilliard MA (2015) EFF-1-mediated regenerative axonal fusion requires components of the apoptotic pathway. Nature 517:219-222.

Nix P, Hisamoto N, Matsumoto K, Bastiani M (2011) Axon regeneration requires coordinate activation of $\mathrm{p} 38$ and JNK MAPK pathways. Proc Natl Acad Sci USA 108:10738-10743.

Palikaras K, Tavernarakis N (2016) Intracellular assessment of ATP levels in Caenorhabditis elegans. Bio Protoc 6:e22048.

Park JS, Höke A (2014) Treadmill exercise induced functional recovery after peripheral nerve repair is associated with increased levels of neurotrophic factors. PLoS One 9:e90245.

Peralta S, Garcia S, Yin HY, Arguello T, Diaz F, Moraes CT (2016) Sustained AMPK activation improves muscle function in a mitochondrial myopathy mouse model by promoting muscle fiber regeneration. Hum Mol Genet 25:3178-3191. 
Pulak RA, Anderson P (1988) Structures of spontaneous deletions in Caenorhabditis elegans. Mol Cell Biol 8:3748-3754.

Rena G, Hardie DG, Pearson ER (2017) The mechanisms of action of metformin. Diabetologia 60:1577-1585.

Richardson CE, Shen K (2019) Neurite development and repair in worms and flies. Annu Rev Neurosci 42:209-226.

Rivera-Brown AM, Frontera WR (2012) Principles of exercise physiology: responses to acute exercise and long-term adaptations to training. PM R 4:797-804.

Sachdeva R, Theisen CC, Ninan V, Twiss JL, Houlé JD (2016) Exercise dependent increase in axon regeneration into peripheral nerve grafts by propriospinal but not sensory neurons after spinal cord injury is associated with modulation of regeneration-associated genes. Exp Neurol 276:72-82.

Sood P, Murthy K, Kumar V, Nonet ML, Menon GI, Koushika SP (2018) Cargo crowding at actin-rich regions along axons causes local traffic jams. Traffic 19:166-181.

Thompson PD, Crouse SF, Goodpaster B, Kelley D, Moyna N, Pescatello L (2001) The acute versus the chronic response to exercise. Med Sci Sports Exerc 33:S438-S445, discussion S452S453.

Truong T, Karlinski ZA, O'Hara C, Cabe M, Kim H, Bakowska JC (2015) Oxidative stress in Caenorhabditis elegans: protective effects of spartin. PLoS One 10:e0130455.

van den Brand R, Mignardot JB, von Zitzewitz J, Le Goff C, Fumeaux $\mathrm{N}$, Wagner F, Capogrosso M, Martin Moraud E, Micera S, Schurch B, Curt A, Carda S, Bloch J, Courtine G (2015) Neuroprosthetic technologies to augment the impact of neurorehabilitation after spinal cord injury. Ann Phys Rehabil Med 58:232-237.

van Hedel HJ, Dietz V (2010) Rehabilitation of locomotion after spinal cord injury. Restor Neurol Neurosci 28:123-134.

van Niekerk EA, Tuszynski MH, Lu P, Dulin JN (2016) Molecular and cellular mechanisms of axonal regeneration after spinal cord injury. Mol Cell Proteomics 15:394-408.
Verdú E, Ceballos D, Vilches JJ, Navarro X (2000) Influence of aging on peripheral nerve function and regeneration. J Peripher Nerv Syst 5:191-208.

Vina J, Sanchis-Gomar F, Martinez-Bello V, Gomez-Cabrera MC (2012) Exercise acts as a drug; the pharmacological benefits of exercise. Br J Pharmacol 167:1-12.

Wagner FB, Mignardot JB, Le Goff-Mignardot CG, Demesmaeker R, Komi S, Capogrosso M, Rowald A, Seáñez I, Caban M, Pirondini E, Vat M, McCracken LA, Heimgartner R, Fodor I, Watrin A, Seguin P, Paoles E, Van Den Keybus K, Eberle G, Schurch B, et al. (2018) Targeted neurotechnology restores walking in humans with spinal cord injury. Nature 563:65-71.

Winder WW, Hardie DG (1996) Inactivation of acetyl-CoA carboxylase and activation of AMP-activated protein kinase in muscle during exercise. Am J Physiol 270:E299-E304.

Yan D, Wu Z, Chisholm AD, Jin Y (2009) The DLK-1 kinase promotes mRNA stability and local translation in $C$. elegans synapses and axon regeneration. Cell 138:1005-1018.

Yang J, Wu Z, Renier N, Simon David J, Uryu K, Park David S, Greer Peter A, Tournier C, Davis Roger J, Tessier-Lavigne M (2015) Pathological axonal death through a MAPK cascade that triggers a local energy deficit. Cell 160:161-176.

Zhang D, Xuan J, Zheng BB, Zhou YL, Lin Y, Wu YS, Zhou YF, Huang YX, Wang Q, Shen LY, Mao C, Wu Y, Wang XY, Tian NF, Xu $H Z$, Zhang XL (2017) Metformin improves functional recovery after spinal cord injury via autophagy flux stimulation. Mol Neurobiol 54:3327-3341.

Zhao M, Klionsky DJ (2011) AMPK-dependent phosphorylation of ULK1 induces autophagy. Cell Metab 13:119-120.

Zhou B, Yu P, Lin MY, Sun T, Chen Y, Sheng ZH (2016) Facilitation of axon regeneration by enhancing mitochondrial transport and rescuing energy deficits. J Cell Biol 214:103-119.

Zong H, Ren JM, Young LH, Pypaert M, Mu J, Birnbaum MJ, Shulman GI (2002) AMP kinase is required for mitochondrial biogenesis in skeletal muscle in response to chronic energy deprivation. Proc Natl Acad Sci USA 99:15983-15987. 